\title{
Spin and Pseudo Spins in Theoretical Chemistry. A Unified View for Superposed and Entangled Quantum Systems
}

\author{
K. Yamaguchi," M. Nakano, H. Nagao ${ }^{\dagger}$ M. Okumura, S. Yamanaka, T. Kawakami, D. Yamaki, \\ M. Nishino, Y. Shigeta, Y. Kitagawa, Y. Takano, M. Takahata, and R. Takeda
}

Department of Chemistry, Graduate School of Science, Osaka Universitw, Tovonaka, Osaka 560-0043, Japan - Department of Computational Science, Faculty of Science, Kanazawa University, Kakuma, Kanazan 920-1192, Japan

Received February 15, 2003

\begin{abstract}
A unified picture for magnetism, superconductivity. quantum optics and other properties of molecule-based materials has been presented on the basis of effective model Hanultonians, where necessary parameter values have been determined by the first principle calculations of cluster models and/or band models. These properties of the matetials are qualitatively discussed on the basis of the spin and pseudo-spin Hamiltonian models. where several quantum operators are expressed by spin variables under the two level approxination. As an example, ab initio broken-symmetry DFT calculations are performed for cyclic magnetic ning constructed of 34 hydrogen atoms in order to obtain effective exchange integrals in the spin Hamiltonian model. The natural orbital analysis of the DFT solution was performed to obtain symmetry-adapted molecular orbitals and their occupation numbers. Several chemical indices such as information entropy and unpaired electron density were calculated on the basis of the occupation numbers to elucidate the spin and pair correlations. and bonding characteristic (kinetic correlation) of this mesoscopic magnetic ring. Both classical and quantum effects for spin alignunents and singlet spin-pair formations are discussed on the basis of the true spin Hamiltonian model in detail. Quantum effects are also discussed in the case of superconductivity, atom optics and quantum optics based on the pseudo spin Hamiltonian models. The coherent and squeezed states of spins, atons and quantum field are discussed to obtain a unified picture for correlation, coherence and decoherence in future materials. Implications of theoretical results are examined in relation to recent experiments on molecule-based materials and molecular design of future molecular soft materials in the intersection area between molecular and biomolecular materials.
\end{abstract}

Key Words : Spin. Pseudo spins. Superposition. Entanglement. Quantum șistems

\section{Introduction}

Past decades electronic. magnetic. optical and other properties of molecule-based materials have been accepted great interest in relation to developments of future quantum devices in nano- and meso-scale dimensions. ${ }^{\text {l-13 }}$ Both experimental and theoretical efforts toward such materials have been carried out extensively. The bottom-up approach from molecule to molecule asemblies is usually employed for realization of molecule-based materials in chemistry. ${ }^{\text {ji-13 }}$ About ten years ago. we have started the specially promoted project entitled in "organometallic conjugation" (during 1994-1997). This is a joint study between experimental and theoretical chemists at our Osaka university for discovery and development of such future nano-scale materials. ${ }^{j+}$ Particularly $\pi$-d conjugated systems have been synthesized and their molecular and solid-state structures have been characterized by the $\mathrm{X}$-ray and other physical tecluniques. Theoretical studies on the $\pi$-d conjugated sy'stems have been succeeded in the special project "Metal-assembled Complexes" during 1998-2002. ${ }^{15}$ Active controls of multifuntional behaviors of the species by chemical and physical procedures have been investigated both experimentally and theoretically.

From the view point of theoretical chemistry the electronelectron interaction plays a more and more important role as system sizes under consideration become more and more small. ${ }^{\text {lic }}$ Probably one-dimensional (ID) finite chain is one of the most interesting examples in this regards. Here. we first examine $a b$ initio spin Hamiltonian model for moleculebased magnets. where the effective exchange interactions are determined by the first-principle broken-sy'mmetry (BS) density functional calculations. ${ }^{17.18}$ As an example. the BS DFT calculations of a magnetic ring consisted of 34 hydrogen atoms is performed to determine the potential energy curves of the lowest - and highest-spin states of the species. and the effective exchange integrals are calculated by the energy difference between them. The natural orbital analy sis of the BS DFT solutions is also carried out to obtain symmetry-adapted (SA) natural molecular mobitals (MO) and their occupation numbers. The information entropy: unpaired electron density and pair-excitation index are expressed by the use of these SA MO and occupation numbers to characterize the spin and pair correlations. and bonding nature of the ring ${ }^{10.20}$ These indices are useful for making bridges between the BS DFT and SA CASCI ${ }^{21.22}$ or CASSCF ${ }^{3.24}$ since the natural orbital concept and its occupation numbers are comunon.

Recently quantum effects in molecule-based materials are one of the central issues in molecular science. particularly in relation to development of nano science and quantum 


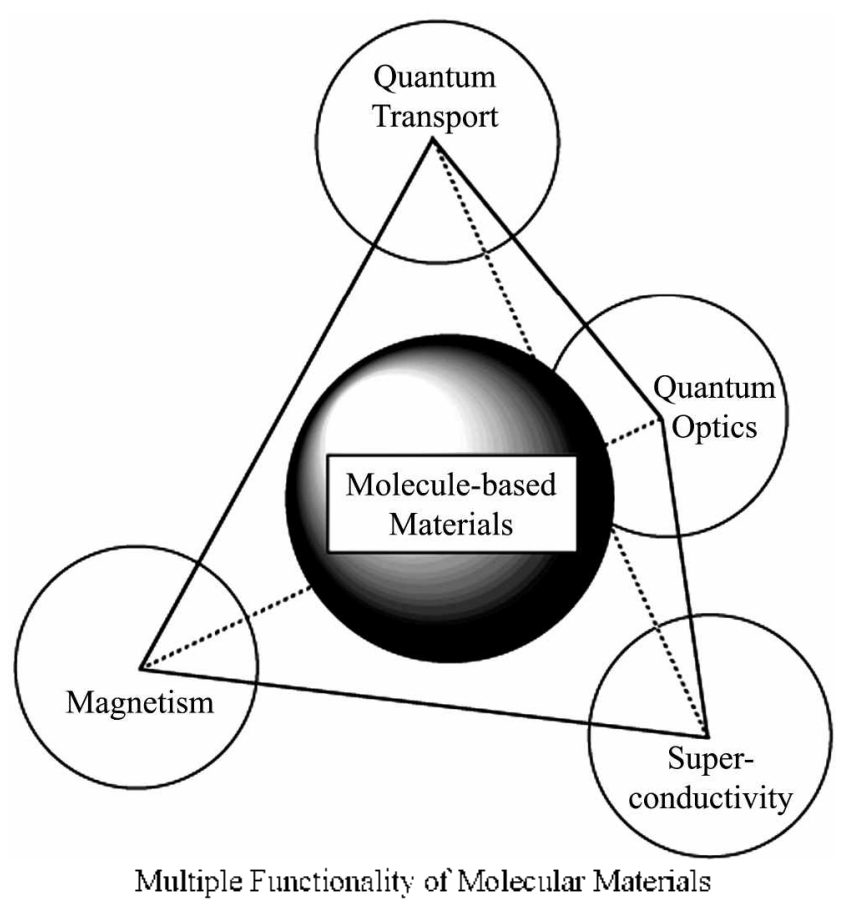

Figure 1. Electronic, magnetic and optical properties of moleculebased materials.

devices. ${ }^{1-9}$ For example. quantum transport of electron. quantum effect for magnetism. quantum fluctuation in high$T_{\mathrm{t}}$ superconductor and quantum optics are current topics in the field of adsanced molecule-based materials as illustrated in Figure 1. So a unified picture for conductivity magnetism. superconductivity and quantum optics is desirable for molecular design of such functional materials. In this paper. effective model Hamiltonians have been introduced in the fields of these topics. and they are rewritten by the pseudo spin Hamiltonians in the case of the simplified two-level model. The real spin Hamiltonian model is examined to elucidate different magnetic behaviors between integer and half-integer spins. ${ }^{35.26}$ The pseudo spin Hamiltonian models are further extended to the superconductivity: ${ }^{27}$ atom optics 28.35 and quantum optics ${ }^{36}$ to elucidate a fomal but common picture among them for molcular design of future materials. Classical long-range orders. coherence and quantum fluctuations violating such orders (decoherence) are discussed from a unified picture. Finally. implications of our recent theoretical results by quantum simulations ${ }^{31-36}$ are discussed in relation to future possibilities of moleculebased materials.

\section{First Principle Calculations}

\section{A. Thoretical Background.}

(i) Magnetic Rings: The first principle calculations of clusters and solids are now feasible to determine various parameters appeared in effective model Hamiltonians for quantum transport. magnetism. super-conductivity and quantum optics for molecule-based materials. However. quantum dýnamics and quantum simulations for phase
The first principle (ab initio) computations

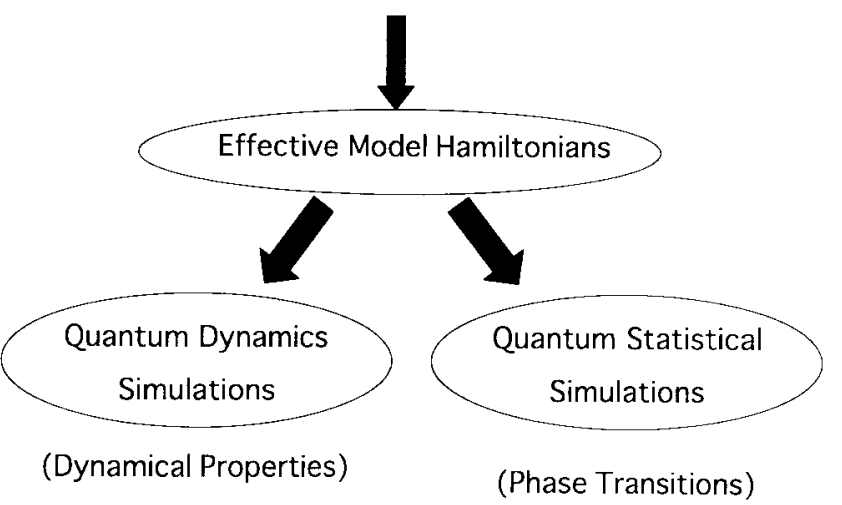

Figure 2. Schematic illustration of theoretical approaches to molecule-based materials.

transitions by the use of ab initio Hamiltonians and wavefunctions are not easy for large systems of our interest even at the present level of computation. Therefore. derivations of reliable effective model Hamiltonians are inevitable before such simulations as illustrated in Figure 2.

The wave nature of electron in ballistic transport in mesoscopic systems ${ }^{1}$ have been well understood because many experimental results have been published recently. The conductance is expressed by the Landauer formula ${ }^{37}$ given by $\mathrm{G}=\left(2 \mathrm{e}^{2} / \mathrm{h}\right)(\mathrm{T} /(1-\mathrm{T}))$, where $\mathrm{T}$ is the transmission probability of electron wave. The universal constant $\left(2 \mathrm{e}^{2} / \mathrm{h}\right)$ is responsible for quantum effect: note that $\mathrm{G}$ becomes infinite at $\mathrm{T}=1$. In this paper, let us start from the other side. namely magnetic insulator. ${ }^{1 \S}$ Magnetic properties of molecule-based materials can be described by the following spin Haniltonian

$$
H_{\text {general }}=-2 J_{x y} \sum_{i}\left(S_{i}^{r} S_{i+1}^{n}+S_{i}^{H} S_{i+1}^{+}\right)-2 J_{i} \sum_{i} S_{i}^{2} S_{j+1}^{*}
$$

where $S_{i}^{\prime \prime}(u=x, y, z)$ denote spin operators at site $i$, and they are expressed by the Pauli matrix as follows.

$$
\begin{aligned}
& 2 S^{r}=\sigma^{r}=\left(\begin{array}{ll}
0 & 1 \\
1 & 0
\end{array}\right), 2 S^{\mathrm{r}}=\sigma^{\mathrm{r}}=\left(\begin{array}{cc}
0 & -i \\
i & 0
\end{array}\right) \\
& 2 S^{s}=\sigma^{*}=\left(\begin{array}{cc}
1 & 0 \\
0 & -1
\end{array}\right)
\end{aligned}
$$

The up- and down- spin states of electron are defined by

$$
|\alpha\rangle=\left(\begin{array}{l}
1 \\
0
\end{array}\right),|\beta\rangle=\left(\begin{array}{l}
0 \\
1
\end{array}\right)
$$

The general spin Hamiltonian in eq. (1) is reduced to the Heisenberg model of $J_{x y}=J_{z}\left(\equiv J_{\mathrm{HB}}\right)$. whereas it becomes the Ising model of $J_{x y}=0$ and $J_{5}\left(\equiv J_{I S}\right) \neq 0$.

The general spin Hamiltonian in eq. (1) is rewritten by

$$
H_{\text {general }}=-J_{x y} \sum_{i}\left(S_{i} S_{i+1}^{-}+S_{i}^{-} S_{i-1}\right)-2 J_{z} \sum_{i} S_{i}^{z} S_{i-1}^{*}
$$


(A)

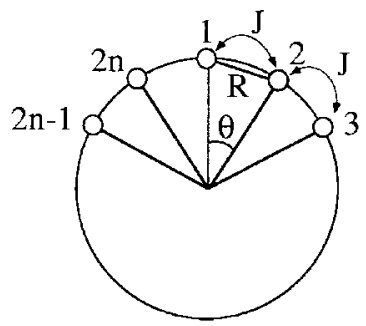

(B)

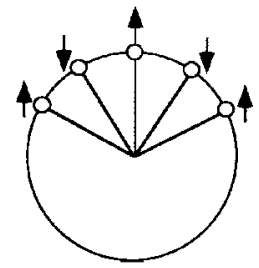

UHF I (LS)

(C)

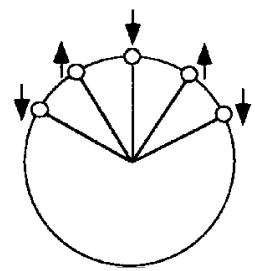

UHF II (LS)

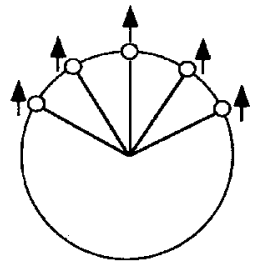

UHF III (HS)

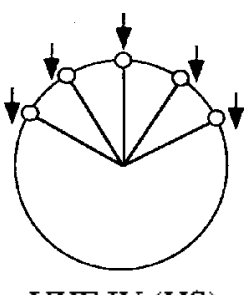

UHF IV (HS)

O: H* $\mathrm{n}=17, \theta=360^{\circ} / 2 \mathrm{n}$

Figure 3. (A) Structure of uniform magnetic chain $(n=17)$, (B) antiferromagnetic (Lowest spin (LS)) and ferromagnetic thighest spin (HS) spin alignments and the contesponding UHF (UDFT) solutions I and III, and (C) their spin inversion structures II and IV.

where $S^{ \pm}$are spin inversion operators defined by

$$
\begin{gathered}
S^{ \pm}=S^{r} \pm i S^{-} \\
S^{-}=\left(\begin{array}{ll}
0 & 1 \\
0 & 0
\end{array}\right), \quad S^{-}=\left(\begin{array}{ll}
0 & 0 \\
1 & 0
\end{array}\right)
\end{gathered}
$$

Then the $J_{x y}$ term is responsible for quantum fluctuation through spin inversions: $S^{+}|\beta>=| \alpha>$ and $\left.S^{-}|\alpha>=| \beta\right\rangle$. On the other hand the $J_{z}$ term induces the spin order responsible for classical spin. In the Ising model at the classical limit $\left(J_{x y}=0\right)$. spins align in the antiparallel manner if $J_{3}<0$. However. two different spin alignments I and II are degenerate in energy as illustrated in Figure 3. This means that spin soliton is generated to destroy the classical spin order (Neel state) in the one-dimensional (ID) chain unless strong anisotropic interaction is operative. On the other hand the quantum fluctuation becomes essential if $J_{x y}<0$. and the resonance between the up-down and down-up spin pairs in the sense of chemistry occurs to give the singlet spin function as

$$
|\Phi(S)\rangle=(|\alpha \beta\rangle-|\beta \alpha\rangle) / \sqrt{2}
$$

The spin Hamiltonian model at the quantum limit $\left(J_{z}=0\right.$ and $J_{x y} \neq 0$ ). which is called as the $\mathrm{XY}$ model. is changed into the free fermion model by the Jordan-Wigner transformation.

and it is solved analytically as in the case of Hückel model $\left(J_{x y}=\beta\right)$. Thus classical and quantum effects in magnetic chains can be described by eq. (1) in a systematic manner.

(ii) Broken-symmetry (BS) Calculations of $J$-Values The effective exchange integrals appeared in the spin Hamiltonian model has been considered to be empirical parameters determined by the experiments. However. recent developments of first-principle computational methods enable us to perform ab intio calculations of $J$ values. For example. broken-symmetry (BS) wavefunctions have been used to calculate the $J$-values in the spin Hamiltonian model. ${ }^{38-42}$ For the purpose the spin-unrestricted HartreeFock (UHF) wavefunctions for the classical spin alignments I and II (see Fig. 3) with the antiferromagnetic order are constructed. respectively. as

$$
\begin{gathered}
\Psi_{J}(\text { UHF I })=\left|\psi_{1} \alpha \psi_{2} \beta \psi_{3} \alpha \ldots \psi_{N} \beta\right| \\
\Psi_{J J}\left(\text { UHF II) }=\left|\psi_{3} \beta \psi_{2} \alpha \psi_{3} \beta \ldots \psi_{N} \alpha\right|\right.
\end{gathered}
$$

These lowest spin (LS) solutions are the eigen functions of spin angular momentum operator $\left(S_{\text {total }}^{2}\right)$ but not that of total spin angular momentum operator $S_{\text {total }}^{2}$. In this sense, these are broken-symmetry (BS) wavefunctions. Then the total energy of the Ising model is formally given by

$$
\begin{aligned}
& E_{I}(U H F I)\left(\equiv E_{I I}(U H F I I)\right) \\
& \quad=\left\langle\Psi_{I}(U H F I)\left|-2 J_{i} \sum_{i} S_{i}^{2} S_{i+1}^{2}\right| \Psi_{J}(U H F I)\right\rangle \\
& =-2 J N S(-S)=2 J_{I} N S^{2}
\end{aligned}
$$

where $N$ is the total site number and $S$ is the size of spin: for example $S^{\prime}=1 / 2$ for hydrogen atom. $H_{I s i n g}$ is the Ising model defined by

$$
\hat{H}_{\text {Ising }}=-2 J=\sum_{i} S_{i}^{2} S_{i+1}^{*}
$$

On the othe hand, the highest spin (HS) UHF solutions are given by

$$
\begin{aligned}
& \Psi_{J I}(U H F I I)=\left|\psi_{1} \alpha \psi_{2} \beta \psi_{3} \alpha \ldots \psi_{N} \alpha\right| \\
& \Psi_{I V}\left(\text { UHF IV) }=\left|\psi_{1} \beta \psi_{2} \beta \psi_{3} \beta \ldots \psi_{N} \beta\right|\right.
\end{aligned}
$$

Then the total energy for the HS aliguments III and IV are given by

$$
\begin{aligned}
& \Psi_{I I I}(U H F H I)\left(\equiv E_{J J}(U H F I V)\right) \\
& \quad=\Psi_{J J}(U H F I I)\left|\hat{H}_{I S n g}\right|\left|\Psi_{J I S}(U H F I I)\right\rangle \\
& =-2 J_{J} N S^{-}
\end{aligned}
$$

Therefore the energy difference between the LS and HS states is formally given by

$$
E_{L S}(U H F L S)-E_{H S}(U H F H S)=4 J_{n} N S^{2}
$$


where LS $=$ I or II and HS = III or IV. The effective exchange integral $\left(J_{z}\right)$ for the Ising model, namely at the classical limit. is obtained by the energy difference between the LS and HS UHF solutions. ${ }^{19.34}$

$$
J_{=}=\frac{E_{L S}(U H F L S)-E_{H S}(U H F H S)}{4 N S^{2}}
$$

The quantum fluctuation becomes important if the orbital overlaps between UHF orbitals are negligible. However the UHF wavefuctions satisfy the following relationship.

$$
\begin{gathered}
\left\langle\Psi_{X}(U H F X)\left|S_{i}^{ \pm}\right| \Psi_{X}(U H F X)\right\rangle=0, \\
(X=\text { I. II, III andIV })
\end{gathered}
$$

This means that quantum fluctuation effect given by the $\mathrm{XY}$ model should be zero if the UHF wavefunction is used as follows.

$$
\left\langle\Psi_{Y Y}(U H F X)\left|\hat{H}_{X Y}\right| \Psi_{Y Y}(U H F X)\right\rangle=0
$$

where the $\mathrm{XY}$ model Hamiltonian is given by

$$
\hat{H}_{X Y}=-J_{x y} \sum_{j}\left(S_{j}^{+} S_{j-1}^{-}+S_{j}^{-} S_{i+1}^{-}\right)
$$

Thus the $J$ values by the general. Heisenberg and Ising spin models are equivalent if the BS UHF solutions are employed for total energy calculations of magnetic chains. The eq. (12) can be applicable to spin-polarized (SP) density functional theory (DFT) ${ }^{43}$ and hybrid DFT (HDFT) solutions because of their similar BS characters to UHF.

(iii) Approximate Spin Projections for UHF and DFT for Mesoscopic Radical Clusters: The symmetry'-adapted $\mathrm{CASCI}^{30.21}$ and $\mathrm{CASSCF}^{23.24}$ calculations are desirable for depicting potential energy curves in all regions of bond dissociation in the magnetic ring. Such treatments are. however very difficult for mesoscopic magnetic clusters. since the number of CAS active orbitals exceeds the current limit (16 orbitals at the present stage). On the other hand. spin projection is easy for the localized spin clusters in the dissociation region (III). since UHF and spin-polarized DFT orbitals for radical sites are essentially orthogonal. Since the total energies of the HS and LS UHF (or DFT) solutions correspond to those of the Heisenberg models. respectively. the energy' gap can be used to estimate the effective exchange integrals $\left(J_{z}\right)$ as shown in eq. (12). The energy gain by the spin projection can be estimated by eq. (12). since $J$ is determined even for larger systems. If the electron correlation is taken into account under the UHF approximation. the effective exchange integrals by UHF in eq. (12) are simply replaced by that of UHF-X (X=MP. CCSD. CCSD (T) $)^{+4}$

On the other hand the spin projection of the UHF-X wavefunctions in intermediate bonding region (II) is a difficult task since the orbital overlaps between radical orbitals are significantly large. The Löwdin-type spinprojection scheme $e^{45}$ is utilized for this purpose. However it often provides incorrect $J$ values because of the introduced approximations. ${ }^{46.47}$ Alternately. approximate spin projections are feasible for UHF-based and spin-polarized DFT methods to calculate potential curves for dissociations from strong bonding region (I) to region III. ${ }^{3 !}$ To this end. we have considered an approximate but size-consistent spin-projection procedure. where the denominator in eq. (12) is modified so as to reproduce the extremal values of the total spin angular momentum in regions I and III as

$$
\begin{aligned}
& \Delta(Z I)={ }^{H S}\langle\hat{S}\rangle(Z)-{ }^{L S}\left\langle S^{2}\right\rangle(Z) \\
& -S_{d} f(N)\left[{ }^{L S}\left\langle S^{2}\right\rangle(Z)-S_{r}\left(S_{r}+1\right)\right]
\end{aligned}
$$

where $f(N)=(N-4) \quad(\mathrm{N}>4$ and even numbers).

and $Z=$ UMP. UCC or DFT. and $S_{r}$ denotes the exact spin angular momentum for the clusters under discussion:

$$
S_{r}=n\left(S_{a}-S_{b}\right) \quad(N=2 n)
$$

where $S_{a}$ and $S_{b}$ are the size of spins at the nearest sites a and $b$ in general. The effective exchange integral for the Heisenberg model by the approximate spin-projected (AP-) UMP. UCC and DFT methods are. therefore. given by

$$
J_{\mathrm{HE}}(A P-Z)=\left[{ }^{H S} E(Z)-{ }^{L S} E(Z)\right] / \Delta(Z I)
$$

The $J_{\mathrm{HE}}\left(. A F^{\prime}-Z\right)$ value almost reduces to that of eq (12) in magnetic region III, while it becomes a theoretical parameter for the spin projection in the intermediate (II) and strong overlap (I) regions, where the spin-contamination effects in UHF and spinpolarized DFT wavefunctions are more or less decreased.

The potential curves by the UMP method often show humps arising from the spin contaminations. The $J_{\mathrm{HP}}(A P-Z)$ values in eq. (18) can be used to improve the shapes of the potential curves by $Z$. as shown previously. ${ }^{43}$ The total energies by $\mathrm{AP}-\mathrm{Z}$ are given by

$$
\begin{aligned}
& { }^{L S} E(A P-Z)={ }^{E S} E(Z)+J_{\mathrm{HE}}(A P-Z) \\
& \times\left[{ }^{L S}\langle\hat{S}\rangle(Z)-S_{r}\left(S_{r}+1\right)\right][1-h(N)]
\end{aligned}
$$

where $h(N)=(N-4) / N \quad(N>4$ and even numbers $)$.

The AP-Z energy reduces to that of RHF-X or ORHF-X $(X=M P, C C)$ in region I. since the second term in eq. (19) disappears. while it becomes equivalent to the unprojected UHF-X energy in region III. As previously shown. ${ }^{4-2}$ the AP$Z$ potential curves are good approximations to those of UNO CASSCF PT2 (CASPT2) in the case of small hydrogen clusters. The energy correction by spin projection should be normalized by the size of the cluster $(N)$ in order to appropriately estimate the projection effect for large $N$.

$$
\begin{aligned}
& \Delta E(A P-Z)={ }^{L S} E(A P-Z)-{ }^{E S} E(Z) / N \\
& \quad=J_{\mathrm{HB}}(A P-Z)\left[{ }^{L S}\left\langle S^{2}\right\rangle(Z)-S_{r}\left(S_{r}+1\right)\right][1-h(N)] / N
\end{aligned}
$$

Since ${ }^{L S}<S^{2}>(Z)$ is approximately proportional to $N$. the 
projection effect of eq. (19) approaches zero if $N$ becomes larger. In fact. for large $N$.

$$
\triangle E(A P-Z) \approx 4 J_{\mathrm{HB}}(A P-Z) / N
$$

Therefore. the spin-projection correction for the LS state decreases along with an increase in the cluster size $(N)$ and the energy correction by the spin projection is negligible if the site number $N$ exceeds a certain limit. ${ }^{15.20}$ Here this behavior is confirmed by using a mesoscopic radical cluster $\mathrm{H}_{34}$.

\section{B. Ab intitio Calculations of $J$ Values for Mesoscopic} Radical Clusters.

(i) Potential Energy Curves and $J$ Values of $\mathbf{H}_{34}$ : As shown in the preceding section. the effective exchange integrals $\left(J_{\mathrm{HB}}\right)$ in the Heisenberg model can be calculated by the difference between the total energies of the highest spin (HS) and lowest spin (LS) states of the clusters in conformity with our AP scheme given by eq. (18). Estimations of the total energies of the lowest spin state by an approximated spin-projected wavefunction are given by eq. (19). It is interesting to draw the potential energy curves of HS. LS. and approximated spin-projected (AP-) LS states of real clusters in order to elucidate the behaviors of the potential energies with size effects of clusters.

Here. a uniform magnetic ring consisted of hydrogen radical cluster (1) with $N=34$ is investigated by $a b$ intio broken-sy'mmetry methods as illustrated in Figure 3. since CAS for 1 is the thirty-four orbital thirty-four electrons $\{34.34\}$. This is an interesting check of our AP BS scheme for practical utility": note that CAS-based methods are really impossible. The highest-spin (HS) and lowest-spin (LS) BS solutions of 1 were first constructed at the UHF level. and their UHF MOs were utilized for successive UB2LYP. UB3LYP and UBLYP calculations. ${ }^{4+5)}$ The total energies of the AP-LS singlet state were calculated by eq. (19).

Figure 4A shows potential energy curves of the LS state of 1 with and without the AP procedure. From Figure 4A. these potential curves by each computational method are most coincident with each other manifesting that the spin projection effect on the potential curves is small for the LS state of 1 as expected from eqs. (15)-(23). The interlydrogen distances $(R)$ at the equilibrium geometry are about $1.0 \AA$ irrespective of the computational methods. while the binding energies $(\Delta E)$ are quite different between UHF and hybrid DFT methods. showing the following order: ${ }^{\mathrm{LS}} \triangle E(\mathrm{UHF}) \ll$ ${ }^{\mathrm{LS}} \Delta E$ (UB2LYP) $<{ }^{\mathrm{LS}} \Delta E$ (UB3LYP) $<{ }^{\mathrm{LS}} \Delta E$ (UBLYP). Judging from many other examples. ${ }^{17-21}$ this tendency is reasonable. On the other hand the potential energies for the HS state of 1 were shown in Figure 4B. All the surfaces were repulsive in nature. in conformity with no covalent bonding between hydrogen atoms and they become insensitive to the computational methods. The repulsive curve is essential for the Bose-Einstein condensation of hydrogen atoms (see later). ${ }^{51}$

The $J$ values for 1 have been calculated by using total energies of the HS and LS BS solutions. Two different computational schemes $J_{Z}$ and $J_{\mathrm{HB}}$ given by eqs. (12) and

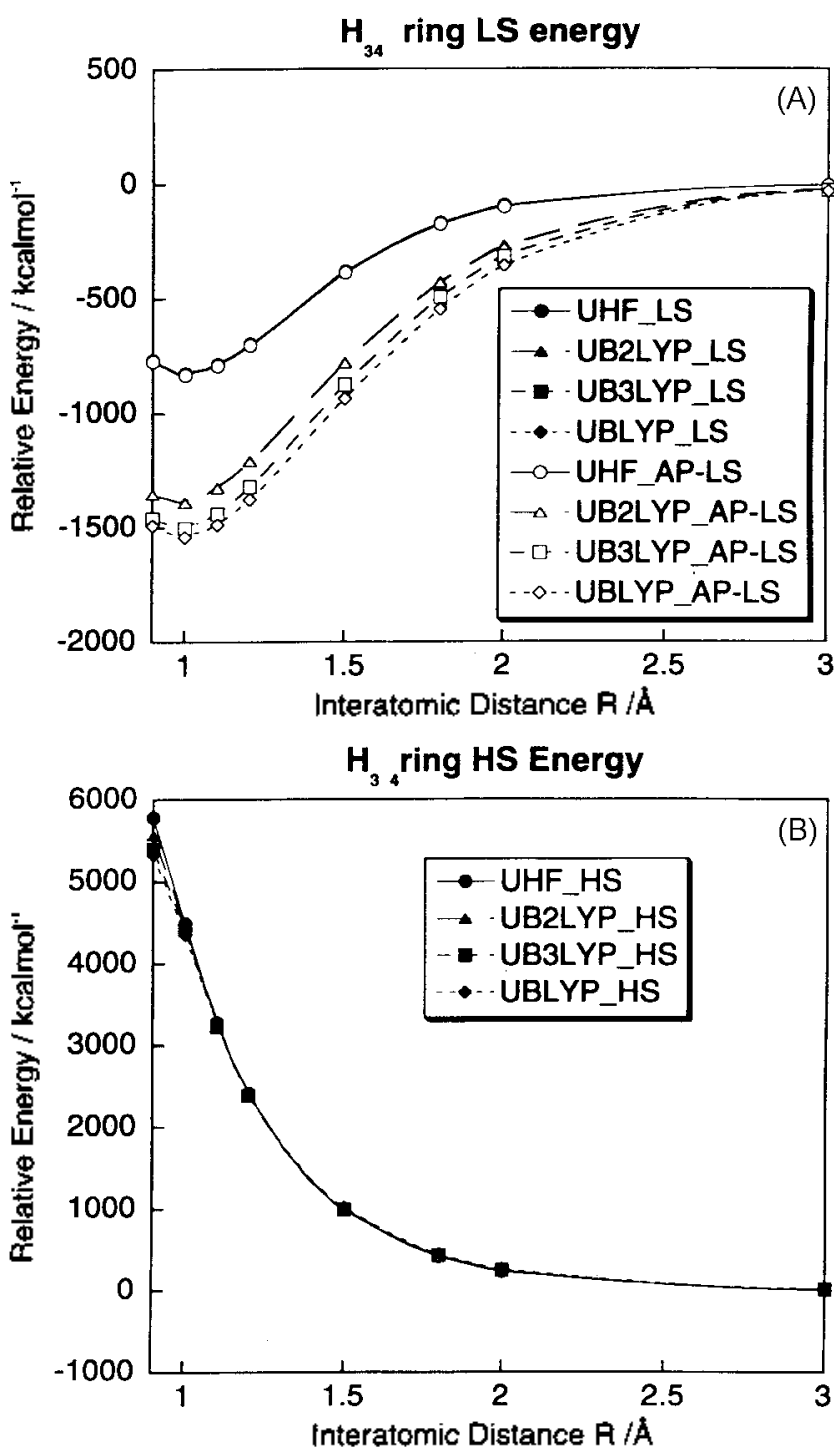

Figure 4 . (A) Total energies of the LS state of the magnetic ring $\mathrm{H}_{34}$ by $\mathrm{UHF}$ and hybrid DFT (HDFT) methods before and atter approsimate spin (AP) projection. The potential curves by each method are almost overlapping, showing no serious correction by AP. (B) Potential curves for the HS state of $\mathrm{H}_{34}$ by UHF and HDFT

(18) have been employed for the purpose. Figure 5 shows variations of $J_{2}$ and $J_{\mathrm{HE}}$, with the change of interatomic distance $(\mathrm{R})$. The spin-unprojected $J_{2}$ and projected $J_{\mathrm{HB}}$ values by each computational method are quite similar in the weak bonding region III $(2.7 \leq R \leq 3.0 \mathrm{~A})$, while they become different in the intermediate region II $(1.8 \leq R \leq 2.5$ $A)$. and they are completely different in the strong overlap regin I $(R \leq 1.8 \AA)$, where the quantum effect is essential.

The $\left|J^{z}\right|$ value by UHF is smaller than those of hy brid DFT methods in accord with general trend in the binding energies. On the other hand. the $\left|J_{\mathrm{HE}}\right|$ values are not so different among the computational methods. since the denominator in eq. (18) becomes small by UHF: note that the ${ }^{L S}<S>$ (UHF) value in eq. (19) is remarkably large because of strong spin polarization.

(ii) Spin Density and Chemical Indices: As shown 


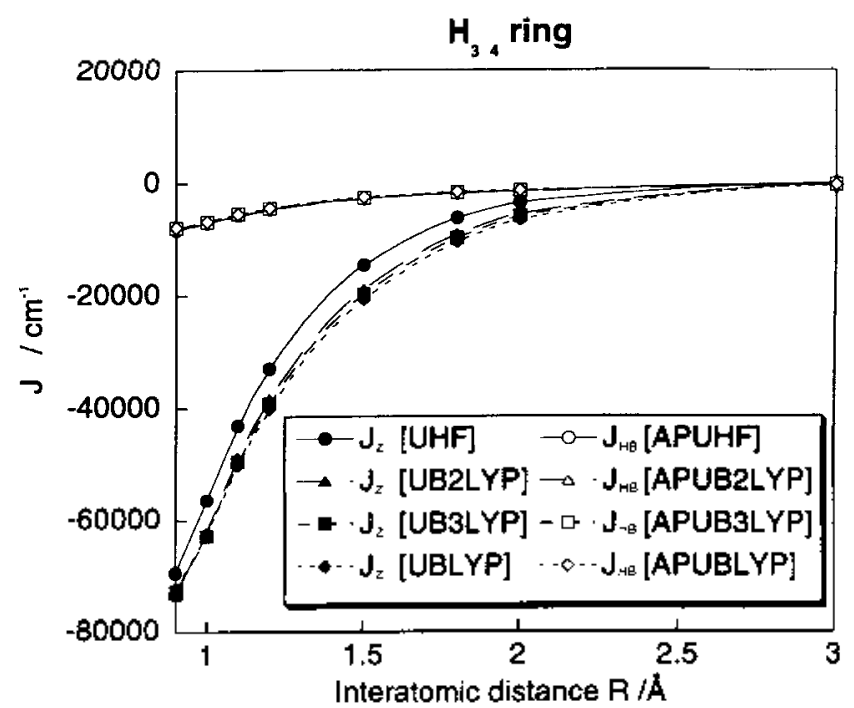

Figure 5 . Variations of the effective exchange integrals $\left(J_{s}\right)$ by UHF and HDFT and those of (THB) after size-consistent AP procedure. The AP procedure is cricial to obtain reasonable J values in the internediate and strong overlap regions.

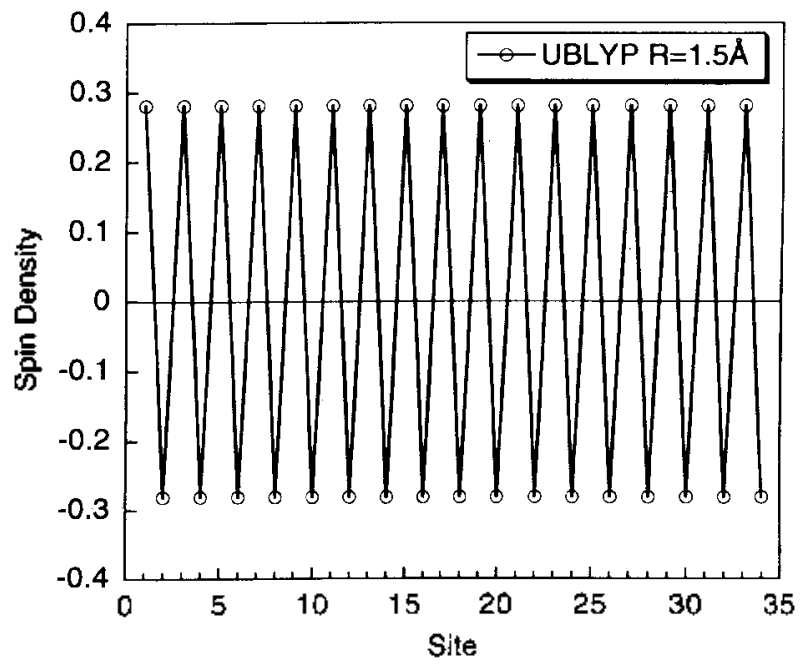

Figure 6. Spin densities on the LS state of the $\mathrm{H}_{3 \downarrow}$ ring at $\mathrm{R}_{\mathrm{H}-\mathrm{H}}$ distance $=1.5 \AA$ by UBLYP, showing the antifenromagnetic spin correlation.

previous- $\quad 1 y^{55.53}$ spin density is a useful index to express inter-site spin correlation function: $K_{\hat{i . j}}=\left\langle S_{\mathrm{S}} \mathrm{S}_{\mathrm{S}}\right\rangle=\left\langle\mathrm{S}_{\mathrm{i}}\right\rangle\left\langle\mathrm{S}_{\mathrm{P}}\right\rangle$ under the classical approximation of spin. Figure 6 illustrates variations of spin densities on hydrogen atoms of 1 at $R=1.5$ A. The up- and down- spin densities apprear alternately: showing an antiferromagnetic spin correlation. The magnitude of atomic spin density by UBLYP is about 0.3 . The spin densities themselves disappear after spin projection familiar in quantum chemistry or because of quantum fluctuation in funite one-dimension system from physical ground. However. it is noteworthy that singlet-ty'pe spin correlation $\left(K_{2}<0\right)$ still survives inspite of such quantum effects. and it is indeed realized if weak three-dimensional interactions are introduced between rings

The on-top spin correlation function is given by $K_{2 . j}=$

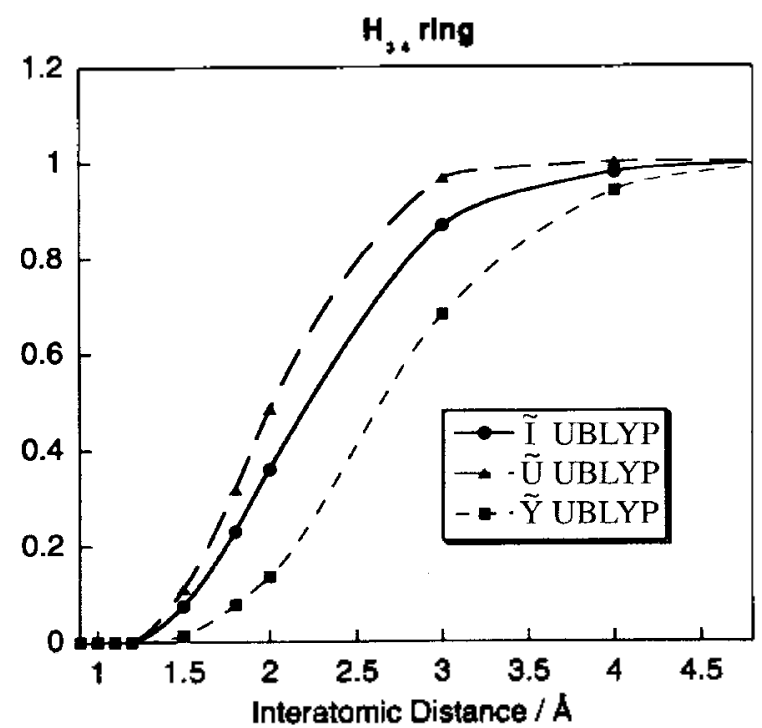

Figure 7. Variations of unpaired electron density $\left(\tilde{U}_{n}\right)$, information entropy $\left(I_{n}\right)$ and pair-excitation index $\left(\tilde{F}_{n}\right)$ for $\mathrm{H}_{3-1}$ ring by UBLYP.

$\left\langle S_{i} S_{S}\right\rangle=\left\langle S_{i}\right\rangle .^{52}$ This value is refered to as unpaired electron density. ${ }^{545 i}$ and expressed by $n_{i}\left(2-n_{i}\right)$. where $n_{i}$ denotes the occupation number of the natural orbitals (NO) of the broken-synmetry UBLYP solution. Then the normalized index $\left(U_{n}\right)$ for unpaired electron density for $2 N$ electron șistem is introduced as

$$
\tilde{U}_{n}=\frac{\sum_{i=1}^{2 N} n_{i}\left(2-n_{i}\right)}{2 N}
$$

where $2 N$ is the maximum number at the strong correlation limit $\left(n_{n}=1, i=1-2 N\right)$. Figure 7 shows variations of $U_{n}$ with $R$. The $U_{n}$ values by the UBLYP calculations increase sharply in the weak and intermediate regions: $1.5 \leq R \leq 2.5$ $A$, showing similar behaviors to those of square values of spin densties. Therefore the $U_{n}$ value is responsible for electron localization or Coulomb hole between opposite spins.

The information entropy ${ }^{57.61}$ by Jaynesis is defined by $-n_{2}$ $\ln n_{i}$, where $n_{i}$ is the occupation number of $\mathrm{NO} i$. Then the information entropy becomes $-2 \ln 2$ for the closed-shell pair $(n=2)$. and the decrease of the bonding character via electron repulsion in the magnetic ring is given by their difference $\left(2 \ln 2-n_{2} \ln n_{i}\right)$ for each pair. The normalized information entropy $\left(I_{n}\right)$ for $2 N$-electron system is defined as

$$
\tilde{I}_{n}=\frac{2 N \ln 2-\sum_{i=1}^{N} n_{l} \ln n_{l}}{2 N \ln 2}
$$

where the maximum (negative) entropy is given by $-2 N \ln 2$ for the closed-shell bonds. Variations of $I_{n}$ with $R$ in Figure 7 indicate that the $\tilde{I}_{n}$ values by UBLYP increase sharply in the intermediate correlation region II $(1.5 \leq R \leq 3.0 \mathrm{~A})$. This is responsible for decrease of the covalent bonds of $\mathrm{H}_{34}$ 
cluster as expected from Figure 4A. The normalized information entropy is therefore a useful index to understand the kinetic energy correlation in complex systems. Further investigation on this point will be given else where.

The pair excitation ${ }^{6]}$ from a doubly occupied NO $i$ to the corresponding vacant $\mathrm{NO}^{*}$ is a possible measure of pair correlation in the real space in analogy with the Cooper pair formation in the $\mathrm{k}$-space for superconductivity. Then the pair excitation index is given by the weight of the doubly excited configuration for each bonding and antibonding NO pair i like the weight $\left(v_{k}^{2}\right)$ of Cooper pair in the BCS wavefunction (see eq. 37). The normalized pair excitation index $\left(\tilde{Y}_{n}\right)$ is introduced to express contribution of doubly excited configuration in each bonding and antibonding natural orbital (NO) pair in the perfect-pairing (PP) type UHF (UNO) and HDFT (DNO) natural orbital CI wavefunction

$$
\tilde{Y}_{n}=\frac{1}{N} \sum_{i=1}^{N} \frac{\left(1-T_{i}\right)^{2}}{1+T_{i}^{2}}=\frac{1}{N} \sum_{i=1}^{N} \frac{n_{i}^{2}-4 n_{i}+4}{n_{l}^{2}-2 n_{i}+2}
$$

where $N$ is responsible for the complete mixing state $\left(T_{i}=0\right.$. $i=1-N$ ) between the ground and doubly excited configurations. As shown in Figure 7 , the $Y_{n}$ values increase gradually with the increase of interatomic distance $R$. The functional behavior of $Y_{n}$ with $R$ is quite different from those of spin density and unpaired electron index $U_{n}$ : note the following tendency: $\tilde{Y}_{n}<\tilde{I}_{n}<\tilde{U}_{n}$ in the whole region of $\mathrm{R}$.

Since $\tilde{U}_{n}$ expresses spin correlations which are expressed by singlet excited configurations constnucted by couplings of bi-triplet excitations and other spin-flip excitations. Figure 7 clearly indicates that the magnetic correlation develops sharply in the course of dissociation of $\mathrm{H}_{34}$ ring. On the other hand the kinetic energy correlation $\left(\tilde{I}_{n}\right)$. which is responsible for covalent bonding. is supressed because of strong electron-electron repulsion. and the pair excitation index is very weak. Thus the broken-symmetry approaches such as hybrid DFT followed by the natural orbital (NO) analysis are sucessfully applicable to elucidate characteristic features of spin. kinetic and pair-excitation correlations in one-dimensional magnetic rings. The three chemical indices. $\tilde{U}_{n}, \tilde{I}_{n}$ and $\tilde{Y}_{n}$ are equally defined for sy'mmetry'-adapted (SA) CASCI and CASSCF wavefunctions. but these wavefunctions are not available for $\mathrm{H}_{34}$. where CAS $\{34$. $34\}$ is cnucial. even at the present level of computer teclunology: Therefore present procedures are practical and handy for theoretical investigations of electronic structures of mesoscopic radical clusters. Our approximate spin projection procedure is also useful to estimate $J_{\mathrm{HB}}$ values for these magnetic materials. Ab initio Heisenberg models with the calculated $J_{\mathrm{HE}}$ can be employed for further quantum statistical treatments of them.

\section{Quantum Dynamics and Phase Transitions}
A. Classical Orders and Coherent Behaviors of Spins.
(i) Quantum Effects for 1D Magnetic Chain: As shown

in section 2. first principle calculations of effective exchange interactions are practically feasible on the basis of the spin polarized hybrid DFT (HDFT) methods. Thus reliable effective model Hamiltonians can be derived from the $a b$ initio computations for molecule-based systents under consideration. Functional behaviors of three different correlation functions within real space are also informative for pictorial understanding of characteristic features of many electron systems with strong electron correlations. As shown in Figure 2. theoretical studies of phase transitions and quantum dynamics are next steps based on the effective model Hamiltonians. Exact quantum-statistical treatments ${ }^{62}$ of them are inevitable to investigate phase transition relating to quantum spin effect and spin flustrations. Here several key concepts are reviewed in relation to our recent quantum simulations.

The spin solid states with classical spin orders described by spin vector models in Figure 8 are realized in the case of three dimensional (3D) lattice systems. In fact. antiferromagnets and ferrimagnets with long-range spin orders can be expressed by the classical spin vector models as shown in I(a) of Figure 8. Past decades. many molecule-based classical (3D) magnets have been synthesized. and their magnetic properties have been summarized in the recent books. ${ }^{14.15}$ On the other hand. spin liquid and spin glass states without longrange order or much more sophisticated states often appear in the cases of $1 \mathrm{D}$ and 2D systems because of quantum

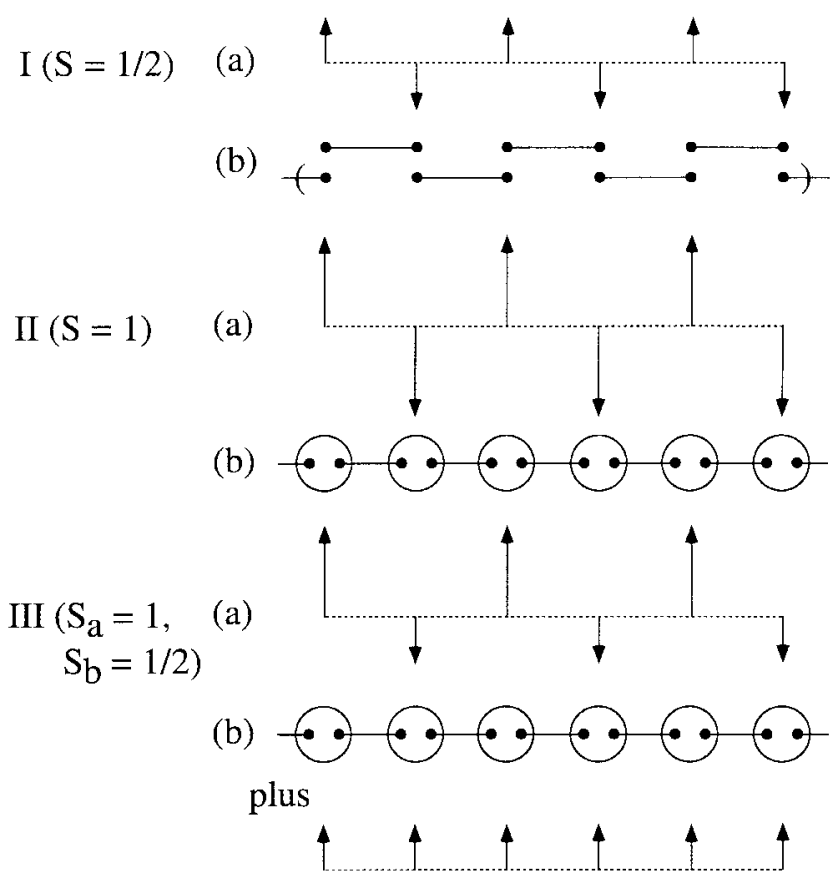

Figure 8. The antiferromagnetic (AF) spin structure for the magnetic chain with spin $S=1 / 2 \quad \mathbf{I}(\mathrm{a})$ and the corresponding quantum spin structure given by the singlet pair bond $\mathbf{I}(b)$ (the other degenerated structure is given in parenthesis), the AF spin structure of $\mathrm{S}=1$ magnetic chain $\Pi$ (a) and the corresponding quantum structure given $b y$ the valence-bond solid (VBS) II(b), ferrimagnetic spin structure with the $S=1$ and $S=1 / 2$ altemating magnetic chain III(a) and the corresponding quantum chain with the $S=I$ quantum chain plus ferromagnetic chain with $S=1 / 2$ III(b) 
fluctuation and/or spin flustration. ${ }^{62.64}$ These states exhibit several quantum coherent phases in general. Theoretical proposals of such low-dimensional (LD) magnets have been performed to obtain possible analogs of the high $T_{\text {. }}$ superconductors. In fact. several LD magnetic systems have been discovered and characterized experimentally. ${ }^{14}$

Haldane ${ }^{25}$ has first pointed out different behaviors of $1 D$ magnetic chains between integer $(S=n)$ and half integer $(S=$ $n+1 / 2$ ) spins. According to his theory the $1 \mathrm{D}$ integer spin chain should have the so-called spin gap. namely the energy: gap between the ground singlet state and the excited magnetic state. This ID sy'stem with Haldane gap has been realized in the case of several $\mathrm{Ni}(\mathrm{II})$ compounds. ${ }^{63}$ Affleck. Kennedy. Lieb and Tasaki (AKLT) ${ }^{-6}$ have explained the Haldane gap by the idea of the valence-bond (VB) solid.

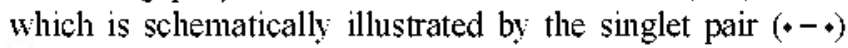
formation between electrons with half integer spin $(S=1 / 2)$. As shown in I of Fig. 8, two different singlet-pair structures are feasible for the ID magnetic chain with $S=1 / 2$, and the resonance between these VB structures occurs even at the zero temperature. On the other hand magnetic sites with $S=$ l spins enclosed by the circle have two hands to make the singlet pairs. leading to the unique solid $\mathrm{VB}$ structure as shown in $\mathrm{II}(\mathrm{b})$ of Figure 8 . Thus the different features between the integer and half integer spin chains can be grasped by the VB concept in chemistry.

The above results suggest many other possibilities of spin alignments $\left(S_{1}, S_{3} . S_{3}, S_{4}\right)$ such as $(1,1,1 / 2,1 / 2)$ and $(1,1 / 2$. 1. 1/2). Classically ferrimagnetic spin alignment is conceivable for both magnetic chains as shown in III(a). While. Yamamoto et al. ${ }^{65.66}$ have shown that the superposed state of the quantum antiferromagnetic state with $S=1$ and the ferromagnetic state with $S=1 / 2$ should appear in the case of quantum spins as shown in III(b) of Figure 8 . The exact diagonalization and DMRG techniques have been used to obtain the heat capacity. $\chi$ T-plot and spin correlation functions for quantum spin systems described by the Heisenberg model. The calculated results have demonstrated the qualitative pictures in Figure 8. Therefore molecular design of new quantum magnets in Figure 8 are interesting and important task for future developments of molecule-based materials. The first principle calculations of these quantities using the ab initio wavefunctions and Hamiltonians are also future targets in quantum chemistry:

(ii) Impurity Effect for 1D Magnetic Chains: Chemical syntheses of LD molecule-based magnets without defects in I of Figure 9 are hard task. Unfortunately, the quantum effects discussed above is sensitive to impurity effects. Eggert and Affleck ${ }^{67}$ have investigated the open chain. where edges cause the inhomogeneity. The existence of the edge state is one of the most interesting properties of the Haldane state in magnetic chain with $S=1$. The singlet and triplet (Kennedy triplet) states become degenerate in the thermodynamic limit. A doping of an impurity of $S=1 / 2$ into the chain brings a local magnetic structure. namely conversion from quantum coherent state to classically spin aligned state.

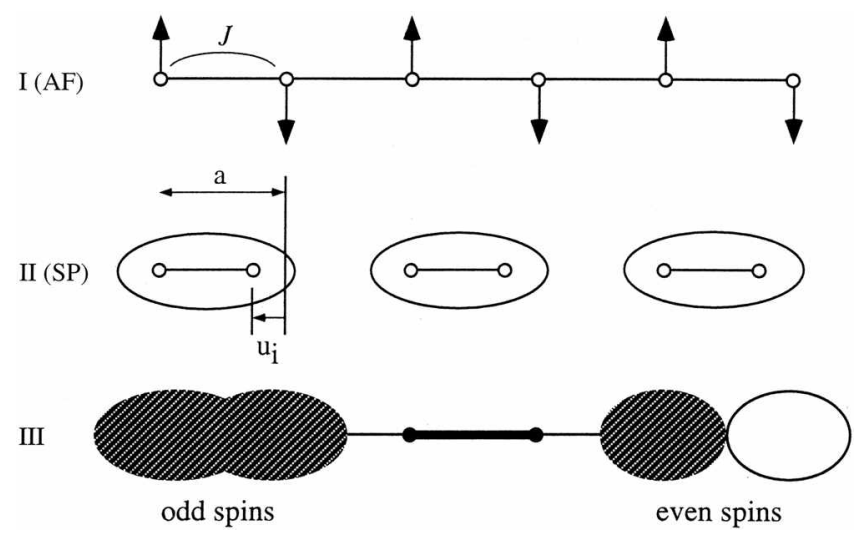

Figure 9. The unitom antiterromagnetic (AF) chain (lattice constant $=$ a) with the exchange integral (I) I(AF), Spin-Peierls (SP) singlet pair with lattice deformation $\left(u_{j}\right)$ II(SP), and the unitom quantum chain with the hond impurity (bold pair). which generates the odd and even spin chains III. The have unitom and inversion altemate atomic spin sllsceptibilities, respectively (see ref. 31 ).

The bond altemation in the magnetic chain often causes the spin Peirles (SP) transition as illustrated in II of Figure 9. The SP state constructed of singlet pair indicated by the circle becomes more stable than the antiferromagnetic (AF) state because of the lattice defomation $t_{i}$. The SP Hamiltonian is given as follows:

$$
\begin{aligned}
H= & -2 J \sum_{j}\left\{1+\lambda\left(\frac{u_{i}}{a}\right)\right\} \boldsymbol{S}_{i} \cdot \boldsymbol{S}_{i+1} \\
& +\frac{K}{2} \sum_{i}\left(u_{i+1}-u_{i}\right)^{2}-\sum h_{i} S_{j}^{2}
\end{aligned}
$$

where $\lambda$ is the spin lattice interaction parameter and $h_{i}$ is the local magnetic field. and a and $K$ denote the lattice and inelastic constants. respectively.

Nishino et $a l^{31.32}$ have elucidated the local magnetic properties of SP systems by investigating the following properties: (1) the local magnetization $m_{i}=\left\langle S_{i}\right\rangle$. (2) spin correlation function $K(i, j)=\left\langle S_{i}^{5} S_{j}\right\rangle$. which is observed by the newtron diffraction teclnique. (3) local susceptibility $\dot{H}_{\text {. }}$. which is responsible for Knight shift of NMR and (4) local field susceptibility $\chi_{i}$ (local). The local susceptibility is introduced to elucidate the local structure in the case of no magnetic moment

$$
\begin{aligned}
\varkappa_{i} & =\frac{\partial}{\partial h}\left\langle S_{i}^{\dot{*}}\right\rangle / h=0 \\
& =\beta \sum_{i}\left\langle S_{j}^{+} S_{i}^{\dot{*}}\right\rangle . \quad(\beta=1 / T)
\end{aligned}
$$

where $h$ is the uniform field. The local susceptibility is nothing but the temperature-dependent spin correlation function. On the other hand. the $\chi_{i}$ (local) value has been investigated as an indication of quantum fluctuation in the study of quantum spin glass. The exact diagonalization and quantum Monte Carlo methods have been used to calculate four indices (1)-(4) at the zero and finite temperatures. 
The quantum Monte Carlo calculations of an open uniform chain with an impurity ${ }^{31.32}$ showed that the strong impurity bond effectively separates the system. and the right and left domains behave almost independently as illustrated in III of Figure 9. The odd chain fragment showed a nodeless shape of $\psi_{i}$ as in the case of the spin density pattern by the BS UHF solution in Figure 6 . while $\psi_{i}$ of even-chain indicated a node. On the other hand, the bond impurity in the bond-alternating chain. namely a defect of alternating order. induces a localized magnetic structure around it.

Renard et $a^{68}{ }^{68}$ have investigated the impurity effect for $\mathrm{CuGe}_{1 . \times} \mathrm{Si}_{x} \mathrm{O}_{3}$ by the neutron diffraction experiment. demonstrating the coexistence of the SP phase and AF phase. though it has been found that $\mathrm{CuGeO}_{3}$ exhibits the SP transition at $14 \mathrm{~K}$. Theoretical studies on the impurity effect on the SP system by Fukuyama et $a .^{60}$ have supported the experiment. This may imply that the impurity in the spin gap systems entails the broken-symmetry state. namely the $\mathrm{AF}$ state. The experimental study on $\mathrm{Cu}_{]_{x}} \mathrm{Zn}_{x} \mathrm{GeO}_{3}$ has elucidated the phase diagrams for the $\mathrm{AF}$ and SP states with the change of $\mathrm{Zn}$ concentration: note that the substitution of $\mathrm{Cu}$ with $\mathrm{Zn}$ corresponds to the hole doping into the magnetic chain.

\section{B. Superconductivity via Spin Hamiltonian model.}

(i) Spin Hamiltonian Model for Superconductivity: One of the central problems in material science is to elucidate the relationship between magnetism and superconductivity in strongly correlated electron șystems. Our group have carried ont several theoretical efforts toward the goal. Here. let us first consider historical backgrounds for superconductivity. Bardeen. Cooper and Schrieffer (BCS) ${ }^{70}$ have presented the theory of superconductivity. The BCS Hamiltonian is defined by

$$
\begin{aligned}
H_{B C S}= & -\sum\left(\varepsilon_{\mathbf{k}}-\varepsilon_{F}\right)\left(1-n_{\mathbf{k}}-n_{-\mathbf{k}}\right) \\
& -\sum V\left(\mathbf{k}, \mathbf{k}^{\prime}\right) c_{\mathbf{k}^{\prime}}^{+} c_{-\mathbf{k}^{\prime}}^{-} c_{-\mathbf{k}} c_{\mathbf{k}}
\end{aligned}
$$

where $\varepsilon_{F}$ is the Fermi level and the second term represents the electron-electron attractive term. and the number density is given by

$$
n_{k}=c_{k}^{+} c_{k}, n_{-k}=c_{-k}^{-} c_{-k}
$$

where $c_{k}^{+}$and $c_{k}$ denote the creation and anihilation operators of electron with the momentun $\mathbf{k}$. Then the total number of electron is given by

$$
N=\sum_{\mathrm{k}}\left(n_{\mathrm{k}}+n_{-\mathrm{k}}\right)
$$

BCS have also introduced the creation and anihilation operators of Cooper pair. namely bosonic electron pair. as

$$
b_{k}^{-}=c_{k}^{+} c_{-k}^{-} \cdot b_{k}=c_{-k} c_{k}
$$

Therefore the interaction term in eq. (29) is given by

$$
b_{k}^{+} b_{k}=c_{k}^{-} c_{-k}^{+} c_{-k} c_{k}
$$

In order to obtain a unified picture of magnetism and superconductivity, let us consider the two level model defined by the pseudo spin Hamiltonian. Anderson ${ }^{27}$ has rewritten the creation and anihilation operators by using Pauli spin matrices as

$$
\begin{aligned}
& 1-n_{\mathrm{k}}-n_{-\mathrm{k}}=\left(\begin{array}{cc}
1 & 0 \\
0 & -1
\end{array}\right)=2 S_{\mathrm{k}}^{-} \\
& b_{\mathrm{k}}^{-}=\left(\begin{array}{cc}
0 & 0 \\
1 & 0
\end{array}\right)=S_{\mathrm{k}^{+}}^{+} b_{\mathrm{k}}=\left(\begin{array}{ll}
0 & 1 \\
0 & 0
\end{array}\right)=S_{\mathrm{k}}^{-}
\end{aligned}
$$

Then the BCS Hamiltonian in eq. (29) is rewritten to the pseudo spin (PS) Hamiltonian as

$$
H_{P S}=-\sum 2\left(\varepsilon_{\mathbf{k}}-\varepsilon_{F}\right) S_{\mathbf{k}}^{-}-\sum_{\mathbf{k} \cdot \mathbf{k}^{\prime}} V\left(\mathbf{k}, \mathbf{k}^{\prime}\right)\left(S_{\mathbf{k}^{\prime}}^{x} S_{\mathbf{k}}^{x}+S_{\mathbf{k}^{\prime \prime}}^{H^{\prime}} S_{\mathbf{k}}^{\mathrm{l}^{\prime}}\right)
$$

where the first and second terms are regarded as the Zeeman and $\mathrm{XY}$ terms respectively. The effective interaction $V\left(\mathbf{k}, \mathbf{k}^{4}\right)$ is given by the effective exchange integral $J$ in the case of $t-J$ model for $2 \mathrm{D} \mathrm{CuO}_{2}$ surface of the high- $T_{c}$ cuprates

$$
\begin{aligned}
& V\left(\mathbf{k}, \mathbf{k}^{\prime}\right)=\frac{J}{N}\left(\cos q^{x}-\sin q^{\mathrm{l}}\right)\left(\mathbf{q}=\mathbf{k}^{\prime}-\mathbf{k}\right) \\
& \Delta_{k}=\Delta_{d}\left(\cos q^{x}-\sin q^{\mathrm{l}}\right)
\end{aligned}
$$

where the $d_{x-1: 2}$ symmetry is assumed for the Cooper pair fomation. Recent calculations by exact diagonalization and quantum Monte Calro technique have shown that the superconducting gap $\Delta_{a}$ is given by 0.1-0.2 J. Then the magnitude of $J$ is a measure of transition temperature for our $J$ model of superconductivity. ${ }^{71}$

Before the discovery of the high $T_{c}$ cuprates by Bednort and Muller, ${ }^{72}$ our UHF calculations of MOM units ${ }^{39}$ have indicated that the magnitude of $J$ value for the CuOCu unit is one-order larger than those of other transition metal oxides. Therefore, after the discovery of the material. we inmediately presented our $J$-model for the high- $T_{c}$ superconductivity ( $T_{c}=\mathrm{cJ}, \mathrm{c}=$ constant) on the basis of the spin fluctuation mechanism. ${ }^{71}$ We have also presented possible moleculebased analogs to the high- $T_{c}$ cuprates on the basis of our $J$ model. To this end. the $a b$ initio calculations of $J$ values for several types of compounds have been carried out. Recently Kawakami et $a l^{73}$ have performed extensive hybrid DFT calculations of $J$ values for several $\pi$-d conjugated sy stems such as (BEDTTTF) $2 \mathrm{X}$ and (BETS $)_{2} \mathrm{X}\left(\mathrm{X}=\mathrm{Cu}(\mathrm{NCS})_{2}\right.$, etc) and demonstrated applicability of the $J$ model to these systems.

The pseudo spin Hamiltonian can be diagonalized by the Bogoliubov transformation to obtain the energy spectrum and $B C S$ wavefunction

$$
\Phi_{B C S}=\prod_{k}\left(u_{k}+v_{k} c_{k}^{+}, c_{-k_{v}}^{-}\right)|0\rangle
$$

where $|0\rangle$ denotes the vacuum, and $u_{n}$ and $v_{k}$ are parameters determined by the variational calculations. Anderson have proposed to express two different states with and without Cooper pair by the up and down pseudo spins.

$$
\alpha=|0\rangle, \beta=c_{k \cdot+}^{-} c_{-k}^{+}|0\rangle=b_{k}^{+}|0\rangle
$$




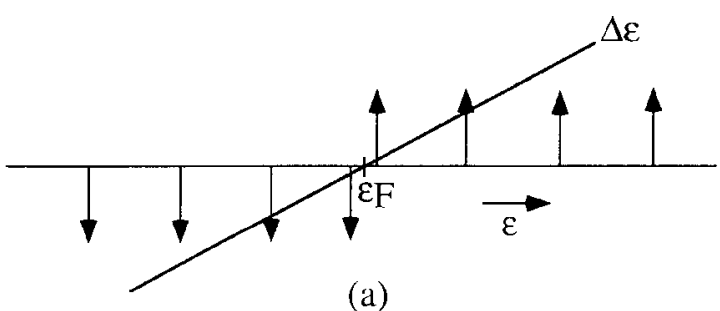

(a)

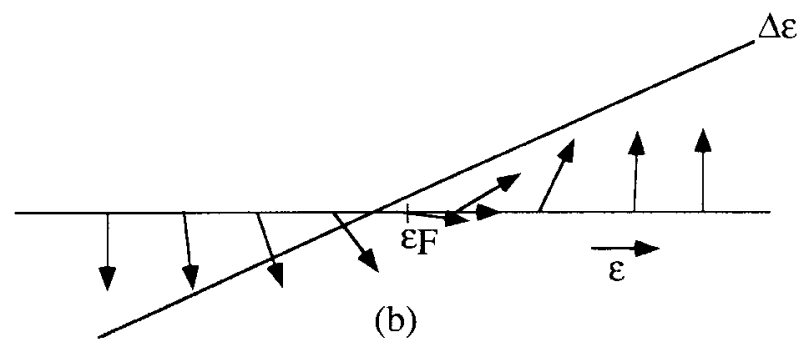

Figure 10. Pseudo spin Hamiltonian models for (a) normal metallic and (b) superconductive states.

Then the BCS wavefunction is rewritten by the pseudo spins as

$$
\Phi_{P S}=\prod_{k}\left(u_{k} \alpha+v_{k} \beta\right)
$$

The superconducting (SC) state is formally regarded as the helical spin density wave (HSDW) state. which is written by the general spin orbital (GSO). $\psi=t_{k} \alpha+v_{k} \beta$. while the normal state is expressed by the uniaxial spins as illustrated in Figure 10. Thus the pseudo spin model is useful for pictorial understanding of the SC state in analogy of the spin aligments. The HSDW spin structures described by GSO have been realized in many spin flustrated magnetic materials. First principle HDFT calculations of these magnetic materials have been carried out by Yamanaka $e t a f .{ }^{74}$ Possibility of the superconductivity in their hole-doped states is also discussed theoretically.

The BCS wavefunction is often rewitten by the bosonic operator as

$$
\begin{aligned}
\Phi_{B C S} & =\left(\prod_{k} u_{k}\right) \prod_{k}\left(1+\frac{v_{k}}{u_{k}} c_{k}^{+} c_{-k_{v}}^{+}\right)|0\rangle \\
& =\left(\prod_{k} u_{k}\right) \exp \left(\sum_{k} a(k) b_{k}^{-}\right)|0\rangle \\
& \propto \exp \left(\sum_{k} a(k) b_{k}^{+}\right)|0\rangle
\end{aligned}
$$

where $a(k)=v_{k} / u_{k}$. This means that the BCS state is regarded as the Bose-Einstein (BE) condensation state of boson. ${ }^{75.76}$ In fact. the BE state for boson with $k=0$ at $T=0$ is defined by

$$
\begin{aligned}
\Phi_{B E} & =e^{-|\phi|^{2}} e^{\phi b^{+} b_{c}^{+}}|0\rangle \\
& =\sum_{n=0}^{\infty} \frac{\phi^{\phi 1}}{n !} e^{-\mid \varphi^{2}=}\left(b_{0}^{+}\right)^{n}|0\rangle
\end{aligned}
$$

where $\left\langle\left\langle\Phi_{B E}\left|b_{i}\right| \Phi_{B E}\right\rangle=\phi\right.$. Then the SC state is regarded
(A)

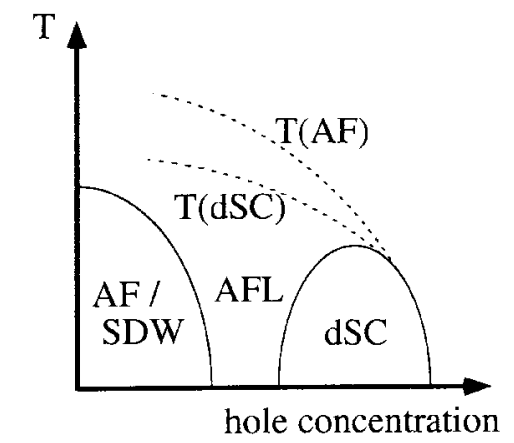

(B)
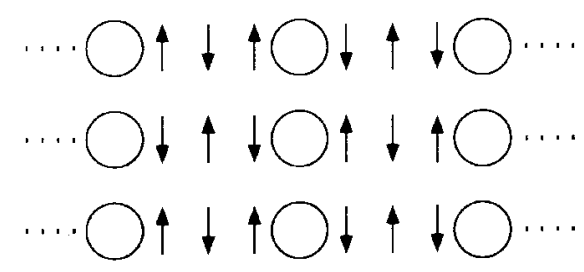

(C)

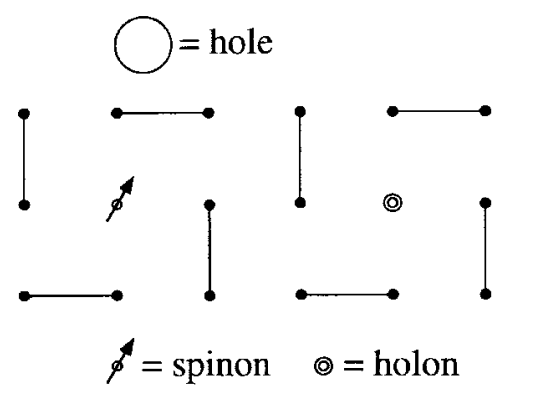

Figure 11. (A) Phase diagram for d-wave superconcuctivity (dSC) of hole-doped cuprates, where AF/SDW denotes the chiral symmetry-broken state, and AFL means the algebraic Fermi liguid (AFL) without symmetry breaking. PG is the psendo gap (or spin gap). (B) stripe structure, where hole and spin have ta and 8a periodic structures, respectively. (C) Spinon and holon generated by hole doping into $\mathrm{CuO}_{2}$ plane of cuprates.

as a phase coherent $\mathrm{BE}$ state with the fixed phase $\phi$. The coherent state has accepted current interest in relation to quantum optics (see below). Recently a phase incoherent state without the long-range order has also attracted as the preformed Cooper pair state in the underdoped cuprates.

(ii) $\mathrm{AF}$ and Superconducting Phases in the 2D Spin Lattice: Anisotropic d- and p-wave superconductivity via electronic mechanism has attracted current interest in relation to the high $T_{c}$ superconductors. Nagao et at $^{77}$ have carried out the theoretical investigations of the high- $T_{c}$ superconductivity from the metallic side (namely from the weak correlation limet) in Figure 11. The temperature Green function techniques have been applied to derive the gap function for superconductivity. and have shown that it becomes attractive because of the change of the SC wavefunction on the Fermi sueface like the $d_{x<y^{2}}$ orbital even if the effective interaction $V\left(\mathbf{k}, \mathbf{k}^{\prime}\right)$ is repulsive in contrast to the s-wave superconductivity via electron-phonon interaction. On the other hand. the high-Tc cuprate superconductors are antiferromagnets before hole doping as illustrated in Figure 11 because of the metal insulator transition in the $\mathrm{CuO}_{2}$ plane via strong electron correlation. ${ }^{71}$ The most important 
effect at the basic microscopic level is to build a large pseudogap of d-wave symmetry in this regard. The pseudo gap is regarded as the spin gap (SG) if the spin fluctuation in the $\mathrm{CuO}_{2}$ plane is predominant. On the other hand the pseudo gap is often considered to be an indication of the preformed Cooper pair before the Bose-Einstein (BE) condensation to the high- $T_{c}$ d-wave superconductivity (dSC). Several groups ${ }^{78-81}$ have pointed out that the stripe shown in Figure 11 may be its origin for psendo gap. Thus three explanations have been presented for the psedo gap in Figure 11(B). Judging from various theories and experiments $^{78-81}$ two-different $S G$ temperatures for the preformed $\mathrm{AF}$ and $\mathrm{ASC}$ ordered states seem feasible as illustrated in Figure 11.

Recently several experimental groups ${ }^{78-81}$ have shown that a magnetically ordered state is induced in the vortex of optimally doped cuprates under the external magnetic field. The STM experiments under the magnetic field have observed the stripe structure with the ta charge density wave (CDW) ( $\mathrm{a}=$ lattice const.). This means that the spin density wave (SDW) with the 8a peridocity is also formed as illustrated in Figure 11, in according with the newtron diffraction experiment. The stripe structure becomes static (classical order) in the case of $\mathrm{Zn}$ doped cuprates because impurities destroy the quantum coherence as shown in Figure 8. Further experimental and theoretical investigations seem necessary for elucidation of roles of stripe and impurity. . $\$-84^{-14}$

C. Atom Optics, Quantum Opties and Quantum Dynamics.

(i) Bose Einstein Condensation of Atoms: The experimental studies on the incoherent and coherent states of Cooper pairs before and after the Bose-Einstein (BE) condensation in eq. 42 are difficult in the high- $T_{c}$ cupurates because several strong interactions are operative. Here. in order to obtain a lucid picture of the BE condensation. let us review recent beautiful discoveries by Cornell. Ketterle and Wieman. ${ }^{28.29}$ They have clearly demonstrated that collections of individual neutral alikali-gas atom ( $\mathrm{Rb}$. $\mathrm{Na} . \mathrm{Li}$ and $\mathrm{H})$ with total number $\mathrm{N}\left(10^{3}<\mathrm{N}<10^{16}\right)$ confined by magneto optical trap (MOT) undergoes the $\mathrm{BE}$ condensation at nanokelvin temperature. Since the momentum of alkali gases has the Bolzman distribution, the thermally averaged de Broglie wave length is given by

$$
\lambda_{t / \mathrm{s}}=\frac{h}{\sqrt{2 \pi M k_{B} T}}
$$

where $M$ is the mass of alkali atom and $k_{B}$ is the Bolzman constant. For example the $\lambda_{t f}$ value of Laser-cooled $\mathrm{Rb}$ atoms at $200 \times 10^{-9} \mathrm{~K}$ is about $4000 \mathrm{~A}$. Namely: the wave length becomes similar to that of visible light at the nanokelvine.

The $\mathrm{BE}$ condensation $(\mathrm{BEC})$ occurs under the condition that the average distance between $\mathrm{Rb}$ atoms is equivalent to $\lambda_{t h}$, namely $\rho=n\left(\lambda_{t f}\right)^{3}=2.612$. where $\rho$ denotes the phasespace density and $n$ is the number density of alkali atom. An important consequence of phase coherence $(\phi)$ for $\mathrm{BE}$

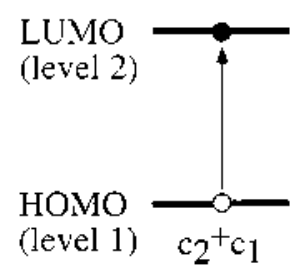

$\left(S^{+}\right)$

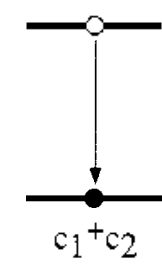

$\left(\mathrm{S}^{-}\right)$

(A)

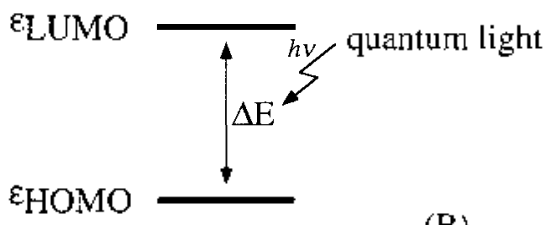

(B)

Figure 12. (A) Jaynes - Cumming model for quantum optics, where excitation operators are expressed by the spin operators and (B) resonance state with quantum light.

condensates in eq. (42) is the occurrence of interference phenomena like light. In fact. Ketterle group ${ }^{28}$ have shown the interference pattern between Laser-cut BE condensates. Thus the Einstein's idea of BEC presented in $1925^{76}$ has been realized in $1995^{28.29}$ This may imply that theoretically possible states should be imagined by a gedanken experiment before experiments and investigated on the basis of quantum simulations.

(ii) Jaynes-Cumming Model for Quantum Optics: The quantum coherence is a central concept in both quantum spins in Figure 8 and atom optics mentioned above. as well as quantum optics. Manipulating of spin and pseudo spins by quantum light would be an interesting field in future. Quantum optical properties of molecules-based materials are therefore very interesting to develop optical computers in future. The quantum optics of these materials is understood in terms of spin Hamiltonian model. the so-called JaynesCumming model..$^{3(1)}$ For simplicity. let us consider the two levels. HOMO (level 1) and LUMO (level 2) of the materials as illustrated in Figure 12.33-3i The electronic excitation from HOMO to LUMO is expressed by $\mathrm{c}_{2}{ }^{+} \mathrm{c}_{1}$ in the second quantized form. while the deexcitation is given by $c_{1}{ }^{-} c_{2}$ Therefore these processes are formally expressed by the spin operators $S^{+}$and $S^{r}$. respectively. While the excited singlet state is defined by $S^{c}$. Then the Hamiltonian of the two-level model for molecule-based materials can be written by the Zeeman term

$$
H_{M}=2\left(\varepsilon_{\text {LIMOO }}-\varepsilon_{\text {HOMO }}\right) s^{z}
$$

where $\varepsilon$ denotes the energy level of $X$ (=HOMO and LUMO). The density matrix for this model is also expressed by the spin operators as

$$
\rho=\left(\begin{array}{ll}
\rho_{11} & \rho_{12} \\
\rho_{\triangle 1} & \rho_{22}
\end{array}\right)=\left(\begin{array}{cc}
4\left\langle S S^{+}\right\rangle & 2\left\langle S^{+}\right\rangle \\
2\left\langle S^{\top}\right\rangle & 4\left\langle S^{-} S^{\top}\right\rangle
\end{array}\right)
$$

Then the inversion population is given by 


$$
\rho_{3 z}-\rho_{11}=4\left(\left\langle S^{-} S^{-}\right\rangle-\left\langle S^{-} S^{-}\right\rangle\right)=4\left\langle S^{-}\right\rangle
$$

The interacting Hamiltonian for two level sy'stem and onemode quantum light is well-known as the Jaynes-Cumming (JC) model ${ }^{3 !}$ as

$$
H_{J K}=H_{M}+H_{L}+H_{I}
$$

where $H_{L}$ and $H_{I}$ mean the Hamiltonian of quantum light and iteraction term, respectively.

$$
H_{L}=\hbar \omega b^{+} b, H_{I}=2 \hbar g\left(b S^{-}+b^{+} S^{-}\right)
$$

It is noteworthy that $b^{+}$and $b$ denote the creation and anihilation operators of light (boson). and $g$ is the interaction parameter. The JC model is therfore regarded as one of the spin boson models. ${ }^{85}$ If $\hbar \omega=\varepsilon_{\text {LAOO }}-\varepsilon_{\text {HOHO }}$, the resonance occurs in this system. The time dependent Schrödinger equation for the JC model can be solved analytically. and the population of the excited state of material and $n$-photon state $\mid n>$ of the field is given by

$$
P_{2, n}(t)=\cos ^{2} \Omega t
$$

where $\Omega$ is the Rabi frequency. This expression is the same for that of macroscopic quantum coherence (MQC) in quantum turneling of single molecule magnets. ${ }^{86.87}$

The coherent state $|\phi\rangle$ of light is expressed by the superposition of $n$-photon states $\mid n>$ (Fock state).

$$
|\phi\rangle=e^{-\mid \phi^{2}: 2} \sum_{n} \frac{\phi^{2}}{(n !)^{1 / 2}}|n\rangle
$$

The two-level system interacting with the coherent light exhibits the quantum collapses and revivals of Rabi oscillation because of the interference effect. ${ }^{3 ! l}$ The intrinsic times for these phenomena are approximately given by

$$
t_{\text {collapse }}=g^{-1} \cdot t_{r e v i v u d}=2 \pi(\bar{n})^{1: t} g^{-1}
$$

where $(\bar{n})^{1: 2}$ is the average broadning of Rabi frequency. The coherent state of light is one of the squeezed states. i.e. Heisenberg's minimum uncertainity state, where quantum fluctuation $\left(\Delta_{1}\right)$ of one of conjugated variables is smaller than the other $\left(\Delta_{-}\right)$. though $\left(\Delta_{1}\right)^{3}\left(\Delta_{2}\right)^{2}=1 / 4$. Several interesting behaviors of two or three level systems interacting with the squeezed light have been found by quantum simulations by Nakano et al. ${ }^{33.36}$

(iii) Bose-Einstein Condensation of Exciton: Past decades there has been great interest in the realization of $\mathrm{BE}$ condensation of excitons in inorganic solids such as $\mathrm{Cu}_{2} \mathrm{O} .{ }^{801}$ Recently several kinds of molecule-based materials have been examined both experimentally and theoretically. in relation to quantum dynamics of excitons. We have also interesting in molecular design of possible organic analognes such as denderimers ${ }^{91}$ to inorganic systems. The ground state of the $\mathrm{BE}$ condensation of excitons is approximately written by the same form as that of $\mathrm{BCS}$,

$$
\Phi_{E X}=\prod_{k}\left(u_{k}+v_{h} e_{k}^{+} h_{-k}^{+}\right)|0\rangle
$$

where $e_{k}^{+}$and $h_{-k}^{+}$denote the creation opetators of electron and hole. respectively. Therefore the Cooper pair for superconductivity in eq. (37) is replaced with the electronhole pair operator. $d_{k}^{+}=e_{k}^{-} h_{-k^{-}}^{-}$. In the low density limit, the coherent state of the ground-state excitons is written by the same equation to eq. (41) for the preformed exciton pair.

$$
\Phi_{E I} \propto \exp \left(a(k) d_{k}^{+}\right)|0\rangle
$$

The density of exciton is in principle tunable by the driving field strength, and therefore optical techniques may explore its behavior at the low density limit as well as the high density limit. where the BCS type coherent state should be realized. However, several relaxation processes are operative in the exciton state of molecule-based materials. and therefore aggregate structures to prevent them are essential for realization of the $\mathrm{BE}$ condensation of excitons. Theoretical studies on several kinds of dendrimers are now in progress in our laboratory. ${ }^{91}$

\section{Active Controls of Quantum States.}

(i) Active Control of Coherent States: Active control of the coherent dynamics of suitable quantun-mechanical systems has become interesting and important in relation to quantum computation and communication. Elements of binary information can be coded in two-stage quantum sy'stems called qubits. Nakanura et ll $^{92}$ have shown that the simplest Josephson-junction qubit is consisted of a small superconductivity island (box) with excess Cooper pair charges connected by a tunneling junction with capacitance and Josephson coupling energy' $\left(f^{2}\right)$ as shown in Figure 13. The superconducting charge box is expressed by a two-state quantum șystem (qubit) with a pseudo spin Hamiltonian

$$
H_{C P B}=-B^{z} S^{z}-B^{x} S^{x}
$$

where $B^{z}$ denotes the charge energy splitting. which is controlled by the gate voltage. It is given by $4 E_{c}\left(1-2 n_{\xi}\right)$. where $E_{c}$ is the single electron charge energy and $n_{g}$ is the gate charge. while $B^{v}=J^{x}$.2. The charge states $n=0$ and $n=1$ are expressed by the up- and down-spins. respectively (see eq. 38)

The Josephson-type effect is also expected for BE condensates of alkali atoms. Recently Leggett et al ${ }^{85}$ have pointed out that the effect is feasible in the system. Generally speaking. after two quantum particles have interacted. they can no longer be described independently of each other. This is called as the entangled state. The nonlocal properties of this state have been clearly demonstrated by violation of

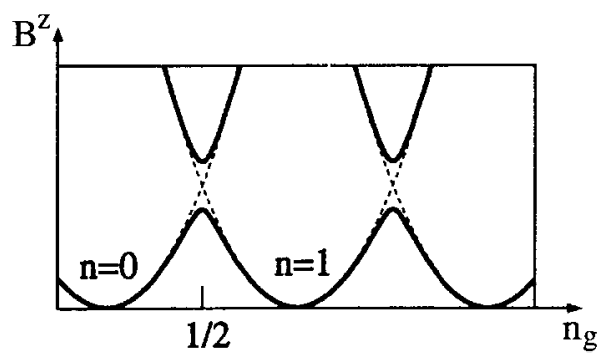

Figure 13. The charging energy of Cooper pair box as a function of the gate charge $\left(\mathrm{ng}_{\mathrm{g}}\right)$ for different number of extra Cooper pairs $\mathrm{n}$. 
Bell's inequalities and Einstein. Podolshy and Rosen (EPR) paradox ${ }^{93}$ However. a mesoscopic superposition of states (Schödinger cat) such as the Josephson junction gets rapidly entangles with its enviromment. losing its quantum coherence. Leggett et af.$^{85}$ have present a spin boson model for such syistems. It models with environment as an oscillator bath coupled to one component of spin. leading to a following Hamiltonian.

$$
H=H_{C P B}+2 S^{2} \sum_{i}\left(\lambda_{i} x_{j}\right)+H_{B}
$$

where $H_{B}$ is the Hamiltonian of the bath and the bath operator couples to $S$ as expressed by the second term. The control of decoherence by environment is an important but difficult problem. Very recently several techniques have been developed for the purpose. ${ }^{94.96}$ The same Hamiltonian has already discussed in the case of quantum tumnelling of spins in single molecule magnets. .7 .08

Raimond. Haroche and collaborators ${ }^{\text {yy }}$ have presented the EPR pair of entangled atoms. and demonstrated the opration of a quantum gate in their microwave cavity quantum electrody'namics experiments. The two particle with singlet spin correlation is formally equivalent to the spin state given by eq. $5:|\Phi(S)\rangle=|\Phi(E P R)\rangle$. This entangled state should maintain the nonlocal singlet spin correlation. However. they have experimentally shown the decay of the coherence. and demonstrated the environment-induced decoherence. which acts faster and faster as the size of the system becomes more and more macroscopic. namely the distance between atoms becomes long. ${ }^{99}$

(ii) Control of Multimode Squeezed Light: As shown in the preceding sections. pseudo spin Hamiltonian models are applicable to diverse physical and chemical systems: (1) real spin particles and magnons. (2) collective two-level atoms. (3) Cooper pairs in superconductors. (4) bilayer quantum Hall sỵstem. (5) Josephson junctions. (6) optical interferometry and (7) light-conmunication network. Several examples discussed above indicate that spin squeezed state i.e. general coherent state. is particularly important in future quantum controls of pseudo spins. Here. let us consider a Sspin sy stem consisting of $2 \mathrm{~S}$ elementary $1 / 2$ spins. ${ }^{10(1)-10: 2}$ The spin function is generally defined by

$$
\begin{gathered}
\psi=c_{\alpha} \alpha+c_{\beta} \beta \\
c_{\alpha \alpha}=e^{j \alpha-\frac{1}{2} \phi} \cos \frac{\theta}{2} . \quad c_{\beta}=e^{j \alpha-\frac{1}{2} \varphi} \sin \frac{\theta}{2}
\end{gathered}
$$

where $\alpha$ denotes the phase factor for Gauge transformation. and $\theta$ and $\varphi$ are polar and azumutual angles in spin space. Therefore the expectation value of spin is given by

$$
\langle\psi|\mathbf{S}| \psi\rangle=(S \sin \theta \cos \varphi, S \sin \theta \sin \varphi . S \cos \theta)
$$

Figure 14 illustrate spin vector in the polar coordinates.

The coherent state of spin is usually defined as the minimum uncertanity state between two noncommuting spin variables. for example $\left(\Delta S_{3}^{2}\right)\left(\Delta S_{i}^{2}\right)=1 / 4$. On the other hand. the quantum fluctuation of one component. $\left\langle\Delta S_{z}\right\rangle$. is

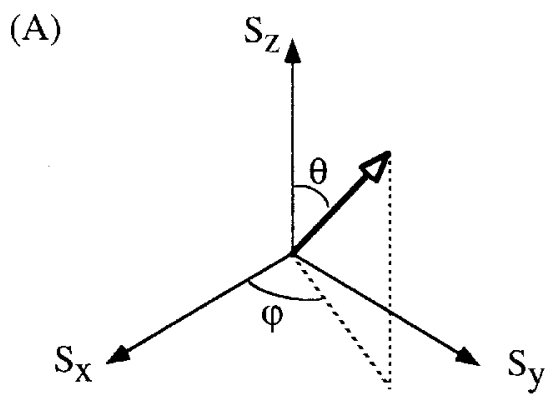

(B)

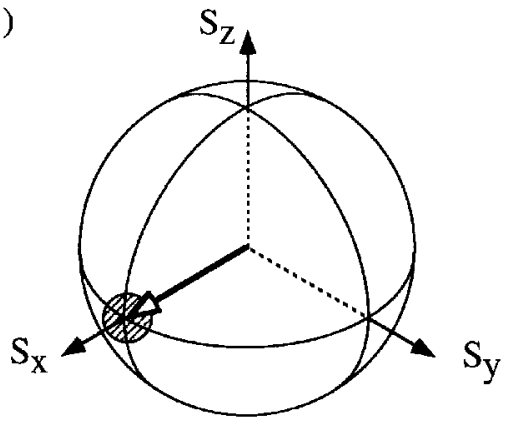

(C)

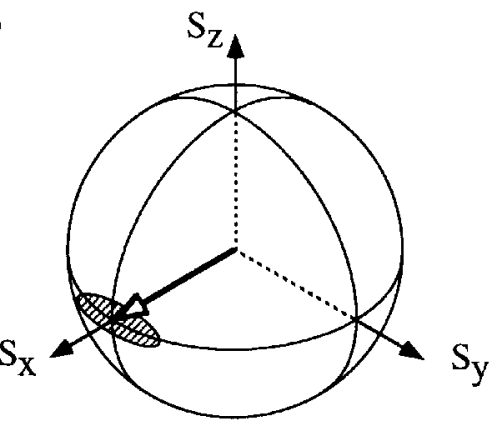

Figure 14. (A) Spin coordinate for spin and pseudo spin $\mathrm{S}_{\text {, (B) spin }}$ coherent state and $(C)$ spin squeezed state.

smaller than that of the other $\left\langle\Delta S_{i}{ }^{2}\right\rangle$ (vice versa) in the spin squeezed state as illustrated in Figure 14. Squeezing of pseudo spins by the use of squeezed fields ${ }^{103.1(14}$ is a current topic in the development of quantum nondemolition measurement ${ }^{105}$ devices and quantum devices. Quantum dynamics simulations of populations and quantum phases have been carried out to elucidate such a possibility by Nakano et al. ${ }^{33-36}$ in our laboratory.

However. the exact definition of quantum mechanical operation for phase is not at all simple task. though the uncertainity relationship $\Delta N \Delta \phi \geq 1 / 2$ is formally derived from the relation $[N, \phi]=i$. Pegg and Burnett $(\mathrm{PB})^{i{ }^{16.1} .107}$ have defined the Hemitian phase operator $\phi$ within the finite subspace $(0 \leq n \leq s)$ of Hilbert space. The PB phase operator works well under the condition that contribution of higher componets $(n>s)$ is small. Therefore the phase operator is closely related to the quantum anomaly problem in general. The expectation value $\langle\phi\rangle$ of phase becomes $\phi$ for colerent light in this definition. in consistent with the experiment. In fact the PB phase operator. ${ }^{1 / 6 i}$ together with quasiprobability distribution function (Q function). ${ }^{88}$ has been successfully used in our recent quantum simulations ${ }^{33-36}$ to elucidate 
dy'namical behavior of the quantum phase properties of the external photon field in atom/molecule/cluster-photon interacting systems. The information entropy ${ }^{57-61}$ has been also utilized to elucidate dýnamics of diagonal (population) and off-diagonal (coherency) molecular densities in these entangled systems. ${ }^{36}$ The computational results have indicated possibility of direct control of quantum coherency by two-mode squeezed quantum field. The multimode squeezed light will be applicable to future optical imaging. opticall parallel processing of information and parallel computing.

\section{Discussions and Concluding Remarks}

A. Effective Model Hamiltonians. The effective exchange integrals in the Heisenberg model have been determined by the first principle spin-polarized hybrid DFT (HDFT) calculations. The spin kinetic and pair correlation functions have been also calculated by using the occupation numbers of natural orbitals of the HDFT solutions. They are useful for systematic analy'sis of developments of these correlation effects in the course of the dissociation process of the $\mathrm{H}_{34}$ ring. Similarly: the Hartree-Fock-Bogoliubov: (HFB) ${ }^{108}$ and Bogoliubov de Genne (BdG) equations ${ }^{109}$ are applicable to elucidate superconducting correlations in mesoscopic systems. ${ }^{110111}$ The first principle HFB and BdG calculations of spin ladder sy'stems have been carried out by Yamaki et $a l^{11}$ to derive an effective model Hamiltonian for superconductivity wa the electron-electron interaction mechanism. The number-density projection ${ }^{113}$ is also necessary to recover the exact number of electron in these broken-sy'mmetry calculations. The equation motion (EOM) calculations ${ }^{11 . .27}$ for stable ground state solutions without instabilities ${ }^{115}$ provide reasonable excitation spectra which is crucial to construct effective model Hamiltonians for quantum optics: ${ }^{116}$ note that EOM/HF (UHF. GHF. HFB) is nothing but the random phase approximation (RPA)/HF (UHF. GHF, HFB) under the mean field approximation. ${ }^{27.117}$ The CAS DFT ${ }^{11}$ by the use of the natural orbitals of UHF solutions (UNO) and unpaired electron density in eq. (24) is also utilized as an alternative to CASPT2. ${ }^{3.24}$ Thus recent developments of the first principle computational methods enable us to determine reliable effective model Hamiltonians in Figure 2.

B. Quantum Simulations. We have discussed quantum effects of spin. atom aggregates. exciton and light in relation to quantum design of molecule-based materials. Several quantum effects discussed here clearly indicate an important role of quantum phase. For example the hole doping of the resonating valence bond (RVB) states consisted of singletpaired spins in the $1 \mathrm{D}$ and 2D systems generates the spinon with one-half spin and holon with charge e. The spinon and holon in the 1D systems are described by the SU(2) and Gaussian conformal field theories with central charge $\mathrm{c}=1$. respectively. ${ }^{\text {(t) }}$ The Lie generators of the theories are given by Virasoro algebra. The charge and spin freedoms are. however. interacting through the Gauge field in the 2D system. ${ }^{119}$ Very recently Takahashi and Aihara ${ }^{120}$ have investigated dynamics of photoexcited states of cuprates using the exact diagonalization teclunique and have shown that time-dependent charge and spin correlation functions exhibit different behaviors, though charge and spin freedoms are coupled. Thus 2D system is really complex. Our previous $a b$ initio calculations of $\mathrm{CuO}$ clusters ${ }^{24}$ indicated the possibily that both charge and spin correlations play important roles for cuprates. leading the electron correlation (EC) mechanism for superconductivity. This may imply that the concept of the Gauge field theory which is popular in elementary particle plysics. play an important role in the physics and chemistry of molecule-based materials in Figure 1.

However. it is noteworthy that the observed $T_{c}$ is largely dependent on the number (s) of $\mathrm{CuO}_{2}$ plane in cuprates. and therefore interband effects (3D effect) are crucial as

$$
T_{c}=-a(s-4)^{2}+b
$$

where $a$ and $b$ are the phenomenological parameters determined by the experiments ( $a=12$ and $b=138 \mathrm{~K}$ ). The micriscopic origin of eq. (59) is considered to be the multibands effect ${ }^{77.84}$ and/or interlayer tunnelling effect confirmed by the Josephson prasuma. which is a coupled wave between Josephson curent and electromagnetic field.

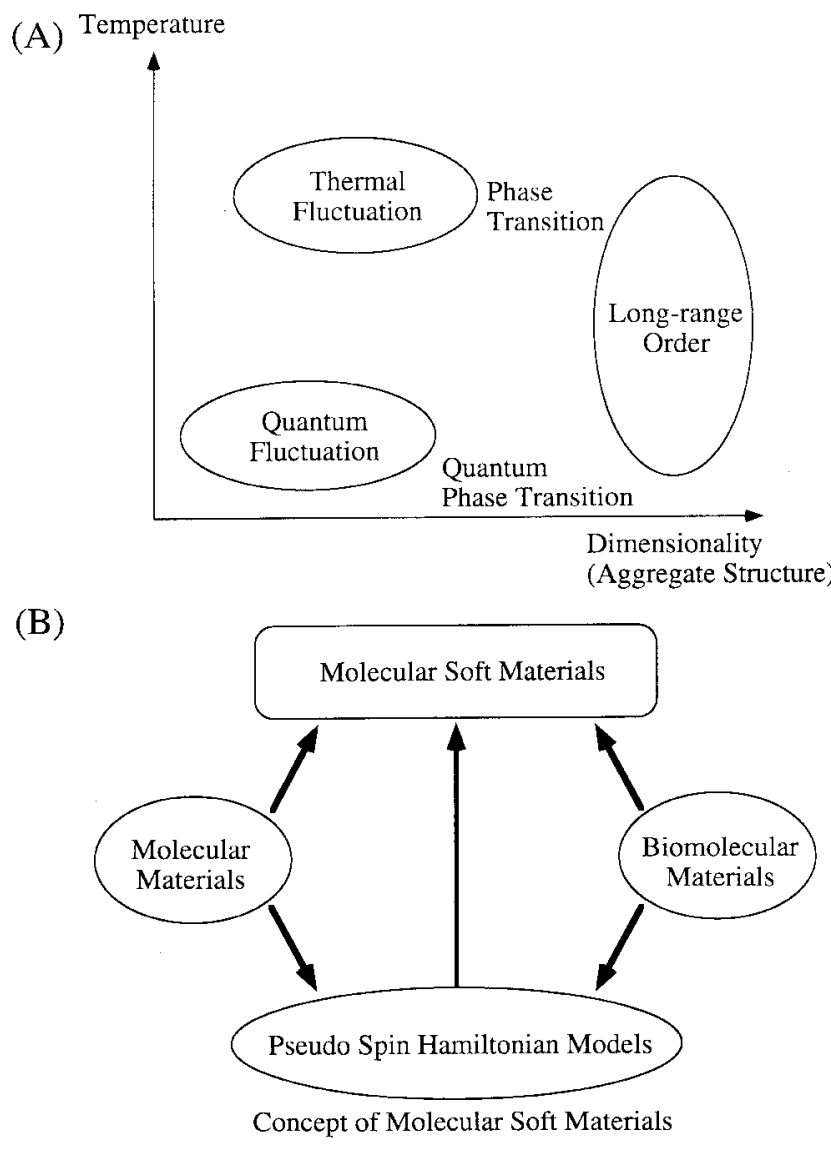

Figure 15. (A) Qauntum and thenmal fluctuations, and related classical and quantum phase transitions, which are highly dependent on lattice dimensionality, namely aggregate structures and temperature, and (B) possible molecular soft materials (see text). 
The quantum dynamics and quantum simulations on the basis of effective model Hamiltonians on the basis of the reliable first principle calculations are also effective for theoretical investigations of complex quantum sy'stems as shown in Figure 2. Both thermal and quantum fluctuations play important roles in future molecule-based materials as illustrated in Figure 15A ${ }^{14}$. 121 The statistical mechanics of a d-dimensional quantum sy'stem at $T=0$ is mapped to $(d+1)$ dimensional classical system with a fake temperature to elucidate quantum and thermal phase transitions. ${ }^{122}$ The $1 \mathrm{D}$ Josephson-junction array in Figure 13 is a typical example to investigate such transitions. Here. relaxation effects to violate coherent states are not touched. though they are extremely important in real systems. Simulation tecluniques are particularly useful to examine these processes since the relaxation parameters are varied in the whole range for which the experiments are not easily accompolished. We feel that quantum effects become more and more important for quantum design of advanced molecule-based materials and the pseudo spin picture presented here is a useful guide for the purpose. . $^{23}$

Recently slow magnetic relaxation has been observed in zero-dimensional (OD) high-spin clusters with strong anisotropy, for example $\mathrm{Mn}_{1 \leq}$ and $\mathrm{Fe}$ sy stems. ${ }^{1: 4}$ and in onedimensional (ID) ferromagnetic or ferrimagnetic chains such as $\mathrm{Co}$ (II)-nitronyl nitroxide nanowires. ${ }^{125}$ The latter ID system can be described by the Ising Hamiltonian in eq (10) because of strong anisotropy' and is referred to as Galuber magnet. ${ }^{1}$ Applicability of these OD and ID molecule-based magnets to quantum computing is theoretically clarfied on the basis of Grover's algorithm under the condition that relaxations such as quantum tunnelling of spin are suppressed. The controlled generation of arbitrary spin superpositions are claimed through the use of multifrequency coherent magnetic radiation in the proposal. ${ }^{127}$ Therefore chemical synthesis of ferri- and ferro-magnetic chains and/or rings with large anisotropy is now an exciting game toward a room-temperature quantum computing. ${ }^{128}$ Here. we did not touch experimental results in detail. They are shown in our other papers. ${ }^{14}$

C. Concluding Remark. Recently an apparent analogy was found between the low-temperature physics of the geometrically frustrated Ising pyrochlore compounds ( $\mathrm{Ho}_{2} \mathrm{Ti}_{2} \mathrm{O}_{7}$, ets) and proton ordering in real ice. ${ }^{139.13 \%}$ These compounds are therefore called as spin ice. Recently biomolecular materials have been accepted great interest. Hydrogen bonding interactions play important roles in these systems. Blinc and Pirc ${ }^{130}$ have shown that the Ising Hamiltonian in eq (10) is formally converted into the memory Hamiltonian if the exchange interaction between spins is rewritten by the hy'drogen bonding interaction: $J_{3}=$ $2 X-1$. where $X=1$ for on-state of hydrogen bonding and $X=0$ for the off-state. They have discussed collective bahavior of hydrogen bonds in proton glasses. We have pointed out an analogy between molecular magnetism and moreculr recognition on the basis of the memory Hamiltonian. ${ }^{131}$ The up- and down-hydroxy groups concerning with
Table 1. Spin and pseudo spin Hamiltonian models for molecular science

\begin{tabular}{cll}
\hline Type & \multicolumn{1}{c}{ Model } & \multicolumn{1}{c}{ Property } \\
\hline 1 & Heisenberg (Ising) model & Magnetism \\
2 & Anderson model & Superconductivity \\
& & Bose Einstein Condensation \\
& & (BEC) of Bosons (Atom, \\
& & Exciton) \\
3 & Jaynes-Cumming model & Quantum Optics \\
4 & Blinc-Pirc model & Glassy ice \\
5 & Memory Hamiltonian model & Molecular recognition \\
\hline
\end{tabular}

the C-C plane such as porphirine ring are regarded as the pseudo up- and down-spins. as shown previously...$^{31} \mathrm{~A}$ genetic algorism developed for complex organic magnets ${ }^{132}$ will be useful for molecular simulations of hydrogen bonding systents. The molecular magnetism and molecular recognition is closely related from the view point of the pseudo spin Hamiltonian model. The quantum effect for proton turnelling might play an important role in specific biological sy'stems such as brain. ${ }^{13.134}$ The intersection area between molecular and biomolecular materials may be developed in future to realize molecular soft materials. which exhibit multiple functions like biological systems as illustrated in Figure 15B.

In conclusion, spin and pseudo spin Hamiltonian models are very useful for a formal but șystematic understanding of magnetism. superconductivity. quantum optics and other properties of hard and soft molecule-based materials as summarized in Table 1. Such a picture is not at all new in physics. but more is different in chemistry ${ }^{135}$ and it may become crucial for development of quantum chemistry for nano- and meso-scopic molecular materials in the intersection area of chemistry . physics and biology: Our efforts during past decades are based on this unified view point. ${ }^{136.137}$ These perspective has been discussed in our recent book. ${ }^{1+}$

Acknowledgment. One of the authors (K.Y.) thank Professor K. Takatsuka for helpful discussions and suggestions. This work has been supported by a Grant-in-Aid for Scientific Research on Priority Areas (14204061) for the Ministry of Education. Science. Sports and Cluture. Japan.

\section{References}

1. Washrum. S: Webb. R. A. Ach: Phs 1986. 35.375

2. Beenakier. C. W. T.: Houten. H. V. Solid State Plns. 1990. H. I.

3. Schonl. G.: Zaikin. A. D. Pln's. Reports 1990. 198. 237.

4. Kastree. M. A. Ren Mod Phus. 1992. 64. 849.

5. Bout. F. A. Phys. Reports 1993. 234. 73.

6. Levy, P. N. Solid State Phys. 1993. 47, 367.

7. Meservey: R.: Tedrow. P. M. Phs. Repots 1994, 238, 173

8. Ferry. D. K.: Grubin. H. L. Solid State Phys. 1995. 49.283

9. Gijs. M. A. M.: Bauer. G. E. W. $A d v$ Phys. 1997. 16. 285.

10. Hecht. S.: Frechet. J. M. Angew. Chom. Int. Ed 2001. 40.74

11. Segura, J. L:- Martin, N. Angew: Chent Int Ed 2001, $40,1372$.

12. Niemeyer C. M. Angen! Chent Int Ed 2001. 40,4128 .

13. Foster, S.; Plantenbers. T. Angew Chent Int Ed 2002. H, 688. 
14. Nakamura, A: Uevama. N.: Yamaguchi. K. Organometallic Conjugation (Kodanshya-Springer. Springer Series in Chemical Phys. 73. 2002)

15. Ito. T. et al. Hetal Assembled Complexes to be published.

16. Yamaguchi, K. Self-Consistent Field Theory and Applications: Carbo, R., Klobuhowski, M., Eds.: Elsevier: Ansterdam. 1990; p 727 .

17. Mitani. M.: Yamaki. D.: Takano. Y:: Kitagawa. Y: Yoshioka. Y: Yamaguchi. K. J. Chem. Phns. 2000. 113. 10486.

18. Yamaguchi. K.: Kawakami. T: Yamaki. D.: Yoshioka. Y. Holecular Aagnetism; Ito. K. Kinoshita. M., Eds.: KodanshaGordon Breach: 2000: $\mathrm{p} 9$ (part I).

19. Kawakami. T: Yamanaka. S.: Takano. Y.: Yoshioka. Y: Yamaguchi. K. Bull. Chem. Soc. Jph. 1998. 71. 2097.

20. Yamaguchi. K: Kawakami. T:: Takano, Y: Kitagawa. Y: Yamashita. Y.: Fujita. H. Iht. J. Quant Chem. 2002. 90.370.

21. Yamaguchi. K: Ohta. K.: Yabushita. S.: Fueno. T. Chem. Phns. Lett. 1977. 19. 555

22. Yamaguchi, K. Int. J. Ouant Chem. 1980. SHA. 269

23. Roos, B. O. Int. J. Outumtum Chem 1980. SHt 175.

24. Andersson. K.: Malmquist P.A.: Roos. B. O. J. Chem. Phns. 1992. 96.1218

25. Haldane. F. D. M. Ptns. Lett. 1983, 934. 464.

26. Affleck. I.: Kemnedy: T.: Lieb. E. H: Tasaki, H. Phws. Rev Lent. $1987,59.799$

27. Anderson. P. W. Phvs Rev 1958. H2. 1900.

28. Ketterle. W. Res Hod Phys. 2002. 74. 1131

29. Cornell. E. A.: Wieman. C. E. Rev. Mod. Phis. 2002. 74. 875

30. Jaynes. E. T.: Cummings. F. W. Proc. IEEE 1963, 51. 100

31. Nishino, M: Ohnishi. H.: Yamaguchi, K: Myashita. S. Phus. Rev: 2000, B62.9463.

32. Nishino, M: Ohnishi. H.: Yamaguchi, K: Myashita. S. Plns. Rev: 2000. B58.9303.

33. Nakano. M.: Yamaguchi. K. Chem. Plys. 2000. 252. 115

34. Nakano. M.: Yamaguchi. K. J. Chem. Plys. 2000. 112. 2769.

35. Nakano, M. Yamaglchi. K. Phws. Rev: 2001. 164033415.

36. Nakano, M.: Yamaguchi. K. J. Chent. Phys. 2002. 116, 10069

37. Landauer R. Foundation of Phvs 1986. 16. 551.

38. Ginsberg. A. P. J. Am. Chem. Soc. 1980. 102. 111

39. Yamaguchi. K.: Takahara. Y: Fueno. T. Applied Otomhm Chem: Smith. V. H. et al.. Eds.: D. Reidel: Boston. MA. 1986: p 155 .

40. Noodleman L: Davidson. E. R. Chem. Phys. 1986. 109. 131

41. Bencini, A.; Totti. F; Doul C. A.: Doclo. K.: Fantuce, P: Barone. V. horg. (Them. 1997.36.5022.

42. Ruiz. E.: Cano. J: Alvarez. S.: Alemany. P. J. Comp. Chem. 1999. 20.1391.

43. Yamaguchi, K. Chent. Phns. Lett 1979.68.477

4.4. Yamanaka, S.: Okumnura. M: Nakano, M: Yamaguchi, K. J. Hol. Struct. Theochem 1994.310, 205.

45. Lowdin. P.-O. Phss. Rev: 1955. 97 . 1494: 1955, 97.1509.

46. Knowles. P. J.: Handy. N. C.J. Chem. Phns. 1988. 88. 6991

47. Schlegel. H. B. J. Chem. Phys. 1986. 84. 4530

48. Becke, A. D. Phws Rev 1988. 438. 3098.

49. Lee C.: Yang. W. Parr R. G. Phrs Rev 1988. B37.785

50. Becke A. D. J Chent. Phns. 1993. 98.5648.

51. Stwalley. W. C.: Nosanow. L. H. Plws. Res. Lett 1976. 36.910.

52. Yamaguchi. K.: Fueno. T. Chem. Phns. 1977, 19.35

53. Yamaguchi. K. Chem. Phss. 1978. 29.117.

54. Takatsuka, K: Fueno, $\dot{T}_{\text {: }}$ Yamaguchi. K. Theor Chime Acto $1978,48.175$

55. Staroverot. V. N.: Davidson, E. R. Chemt. Phns. Lett. 2000, 330. 161 .

56. Staroverov. V. N.: Davidson. E. R. J. Am. Chem. Soc. 2000. 122. 7377.

57. Welri. A. Rev Mod Phvs. 1978, 50,221

58. Shaminon, C. E. Bell. Sist. Tech 1948, 27.379

59. Tannes, E.: Probability Statics and Statistical Phosics:
Rosencrants, R.. Ed.: Reidel. 1993.

60. Collins, D. M. Z. Naturforsch 1993. +8.4. 68 .

61. Yamaguchi. K. Chem. Plys. Lett 1975. 35. 230.

62. Fradkitn. E. Field Theories of Condensed M Hatter Systems. Addison Wesey: Reading. MA. 1991.

63. Moleculor Magnetism; Ito. K.: Kinoshita. M., Eds.: Kodanshya. 1999.

64. Fukui. T: Kawakami. N. Phs. Rev 1997. B55. 14709

65. Brehmer. S.: Mikeska. H.-T.: Yamamoto. S. J. Phus. Condens. Matter: 1997.9.3921.

66. Yamamoto. S.: Brehmer. S.: Mikeskia. H.-T. Phus. Rev 1998. B57. 13610 .

67. Eggert. S.: Aftleck, I. Phos Rev: Lett. 1995, 75, 934.

68. Regnault, L. P.; Renard. J. P.: Dhalenne. G.: Revcolevschi, A Europhys. Lett 1995.32 .579$.

69. Fukuyama. H.: Tanimoto. T.: Saito. M. J. Pln. Soc. Jpn. 1996. 65. 1182

70. Bardeen. J.; Cooper. L. N.: Schrieffer. J. R. Phw Rer: $1957,108$. 1175 .

71. Yamaguchi. K.: Takahara. Y.: Fueno. T; Nasu, K. J. Appl. Phns 1987. 26. L136?

72. Bedtort. T. G.: Muller. K. A. Z. Phs. 1986. 6ł. 189

73. Kawakami. T: Taniguchi. T.: Kitagawa. Y.: Takano. Y.: Nagao. $\mathrm{H}$; Yamaguehi, $\mathrm{K}$. Mfol Phis. 2002, 100. 2641

74. Yamanaka. S.; Yamaki. D; Shigeta, Y; Nagao. H.: Yoshioka, Y.: Suzuki. N.: Yamaguchi. K. Iht. J. Otant. Chem. 2000, 80,664

75. Bose. S. N. Z. Phus. 1924. 26. 178.

76. Einstein. A. Phvs. 1/ath Kl Bericht 3. 18: Situngsber. Press: Akad. Wiss. 1925

77. Nagao. H.: Nishino. M.: Shigeta. Y; Yoshioka. Y; Yamaguchi, K. J. Chent. Phos $2000,113.11237$.

78. Katano. S.: Sato, M. Yamada, K: Suzuki. T: Fukase. T. Phws Rev 2000. B62. Rl4677.

79. Lake. B.: Aeppli. G.: Clausen. K. N.: McMorrow: D. F.: Lefmant1. K.: Hussey. N. E.: Mangkorntong. N.: Nohara. M. Takagi. H: Mason. T. E; Schroder, A. Science 2001, 291, 1759.

80. Lake. B.: Ronnow, H. M: Christensen. N. B.: Aeppli. G.: Lefmann. K; McMorrow. D. F.: Vorderwisch, P. Smeibidl, P.: Mangkorntong. N.: Sasagawa. T:: Nohara. M.: Takagi. H.: Mason1. T. E. Natwe 2002. H15. 299

81. Hoftuman. J. E.: Hudsont. E. W.: Lang. K. M.: Madhavan. V: Eisaki, H.: Uchida. S.: Davis. J. C. Science 2002. 295. 466.

82. Franz. M.; Tesanovic. Z.: Valek, O. Phis. Rev 2002. $566,054535$.

83. Herbut, I. F. Phus, Rev: $2002, B 66.094504$

84. Yamaguchi. K. Int J. Ouant Chem 1990.37. 167

85. Leggett. A. J. Rev . Hod. Phys. 2001. 73. 307.

86. Yamaguchi. K.: Yamanaka. S.: Nishino. M.: Takano. Y.: Kitagawa. Y.: Nagao. H: Yoshioka. Y. Theor. Chem. Ace. 1999, 102. 328.

87. Nagao. H.: Nishino. M.: Shigeta. Y; Soda, T.: Kitagawa, Y.: Onishi, T: Yoshioka, Y.: Yamaguehi. K. Coord. Chem. Rev 2000. 198. 265.

88. Walls. D. F.: Milburtr. G. T. Quantum Optics. Springer-Verlag: Berlin. 1994

89. Lin. J. L.: Wolte, J. P. Phys. Rev: Lett. 1993, 71. 1222.

90. O'Hara. K. E.: Suilleabhain. L. O.: Wolfe. J. P. Phrs Rev 1999. $B 60.10565$.

91. Nakano. M.: Fujita. H.: Takahata. M: Yamaguchi. K. J. Am. Chem. Soc. 2002. 124. 9648

92. Nakamura. Y.: Pashkin. Yu. A.: Tsai. J. S. Natme 1999. 398. 786.

93. Scully, M. O.; Zubairy, M. S. Otamtum Optics. Cambridge University Press: 1997: Chapter 18.

94. van der Wal. C. H.: ter Haar. A. C. T.: Wilhelm. F. K.: Schouten. R. N.: Harmans. C. T. P. M.: Orlando. T. P.: Lloyd. S.: Mooij. .T. E. Science 2000. 290.773

95. Friedman. J. R.: Patel. V.: Chen. W.: Tolpygo. S. K.: Lukens, J. E Nature 2000. +06.43

96. Yu. Y. Han, S.; Chu. X.: Chu, S.: Wang. Z. Science 2002. 296. 
889

97. Garcia-Pablos. D.: Garcia. N.: Raedt. H. De. Phys. Rev 1997. B55. 937.

98. Raedt. H. De.: Mivashita. S.: Saito. K.: Garcia-Pablos. D.: Garcia, N. Phts. Rev 1997, B56. 11761

99. Rainmond J. M.: Brune. M.: Harosche, S. Rev Mod Phvs. 2001. 73. 365 .

100. Kitagawa. M.: Ueda. M. Phns. Ren. 1993. A47. 5138.

101. Nakakima. T.: Aoki. H. Phis. Res: 1997. B56. R15550.

102. Sorensen. A.: Molmer. K. Pln's. Rev: Lett. 1999. 85. 2274.

103. Zhang. W.-M.: Feng. Da. H.: Gilnore. R. Rev Mfod Phns 1990. 62,867 .

104. Kolobov. M. I. Ret Mod. Phss. 1999, 71, 1539.

105. Braginshy. V. B.: Khalili. F. Ya. Res. Mod. Phys. 1996. 68. 1.

106. Pegg. D. T.: Barnett. S. M. Phys. Rev: 1989. A39. 1665.

107. Lynch. R. Ptns. Rep. 1995. 256. 367.

108. Bogoliubov. N. N. Sor. Phns. JETP 1958. 7.41.

109. de Gemes. P. G. Superconductivity of Metals and Allows: Benjamin: New: York: 1966.

110. Marumori, T. Prog Theoret Phys 1960. 2t.331.

111. Yamaguchi. K: Yamaki. D.: Kitagawa. Y.: Takahata. M. Kawakami. T.: Osaku. T.: Nagao. H. Iht. J. Qtomt. Chem. in press.

112. Yamaki, D. et at to be publised.

113. Peirls, R. E: Yoccoz. J. Proc. Phys. Soc. 1987, 70, 381.

114. Sawada. K. Phws. Rev 1957. 106. 372.

115. Rowe. D. J. Aitcl. Ptns. 1966. 80.209

116. Thouless. D. J. Aucl Phys. 1960.21.225.

117. Yamaki. D.: Shigeta. Y: Yamanaka. S.: Nagao. H.: Yamaguchi. K. Int. J. Otount Chem. $2000,80,701$.

118. Takeda, R.: Yamanaka. S.: Yamaguchi, K. Chem. Phys. Lett. $2002,366.321$

119. Nagaosa. N.: Lee. P. A. Phns. Ren. 1992. B4. 966.
120. Takiahashi. A.: Gomi. H.: Aihara, M. Phis. Rer: Lett. 2002. 89 206402.

121. Gatteschi. D: Yamaguchi. K. Holecular Hagletisn: From Molecular Assemblies to the Devices' Coronado. E. at al. Eds.: Kluwer. Academic Pub.: Netherland. 1996: p 561.

122. Sondhi. S. L.; Girvin. S. M:- Carini, J. P.; Shahar. D. Rev: Mod Phrs 1997,69.315

123. Sny der. J.: Slusky, J. S.; Cava. R:; Schfer. P. Natue 2001, +13 48

124. Gatteschi. D.: Caneshi. A.: Pardi. L.: Sessoli. R. Science 1994. 265. 1054

125. Caneschi, A.: Gatteschi, D.: Lalioti, N.: Sangregorio. C:- Sessoli. R; Venturi. G.; Vindigni. A.: Rettori, A.; Pini. M. G.: Novak. M. A. Angew Chem. Int Ed 2001, f0. 1760.

126. Gauber. R. I. J. Hath. Phus. 1963. +. 294.

127. Lenenberger. M. N.: Loss. D. N. Natwe 2001. 110.789.

128. Yamashita. M. et al . . Am. Chem. Soc in press.

129. den Hertog. B. C.: Gingras. M. J. P. Phys. Rev: Lett. 2000, 84. 343.

130. Bline, R; Pire. R. Theoretical Treatntents of Hydogen Bonding, Hadzi. D.. Ed.: Tohn-Wiley: 1997.

131. Yamaguchi. Y. Functionality of M foleculor Systems: Nagakura. S.. Ed.: Springer: Tokyo. 1998: vol l. p 67.

132. Oda. A.: Nagao, H.; Kitagawa, Y.: Shigeta Y: Yamaguchi, K. Int. J. Outh. Chent $2000,80.646$.

133. Theumann. W. K.: Erichsenm. R. Jr. Phos. Ren: 2001, E6t. 061902 .

134. Ibu. M.: Pribram. K. H.: Yasue. K. Int. J. Mod Phus. 1996. B10. 1735 .

135. Anderson. P. W. Science 1972.177, 393.

136. Yamaguchi. K.: Fueno, T. Kagaka 1986. H. 372 (in Japanese).

137. Yamaguchi, K. Self Consistent Field, Theory and Applications: Carbo. R.. Klobukowski. M.. Eds.: Elsevier: 1990. 